\title{
A Temperature-Insensitive FBG Acceleration Sensor with Sinusoid-Shaped Curved Beams
}

\author{
Li Hong \\ Institute of Disaster Prevention \\ Yuzi Zhang \\ Institute of Disaster Prevention \\ Ruwang Mu \\ Institute of Disaster Prevention \\ Yanan Li \\ Institute of Disaster Prevention \\ Zhongchao Qiu ( $\nabla$ qiuzc1987@163.com ) \\ Institute of Geophysics
}

\section{Research Article}

Keywords: Fiber Bragg Grating (FBG), chirp effect, temperature-insensitive, acceleration sensor

Posted Date: November 25th, 2020

DOl: https://doi.org/10.21203/rs.3.rs-107936/v1

License: (c) (1) This work is licensed under a Creative Commons Attribution 4.0 International License.

Read Full License 


\section{A Temperature-Insensitive FBG Acceleration Sensor with}

\section{Sinusoid-Shaped Curved Beams}

Li Hong ${ }^{1}$, Yuzi Zhang ${ }^{1}$, Ruwang $\mathrm{Mu}^{1}$, Yanan $\mathrm{Li}^{1}$, Zhongchao Qiu ${ }^{1,2} \bowtie$

( ${ }^{1}$ Institute of Disaster Prevention, Sanhe 101601, China; ${ }^{2}$ Institute of Geophysics, Beijing 100081, China)

Abstract: It is an important means to identify and evaluate the structural damage of buildings before and after earthquake disaster by using fiber Bragg grating acceleration sensor to obtain the natural frequency of building structure.

In order to solve the problem of temperature cross sensitivity of fiber Bragg grating $(\mathrm{FBG})$ in structural health monitoring, a novel acceleration sensor based on strain chirp effect which is insensitive to temperature is proposed. A kind of M-shaped double cantilever beam structure is developed. The fiber grating is pasted in the gradient strain region of the beam, and the chirp effect is produced under the action of non-uniform stress, and then the change of acceleration is converted into the change of reflection bandwidth to demodulate and eliminate the temperature interference. Through theoretical analysis, simulation and experimental verification with rectangular beam sensor. The results show that the sinusoidal curvature beam sensor is insensitive to the change of temperature and is more likely to produce chirp effect. The sensitivity is about $317 \mathrm{pm} / \mathrm{g}$, and the natural frequency is $56 \mathrm{~Hz}$.

Keywords: Fiber Bragg Grating (FBG); chirp effect; temperature-insensitive; acceleration sensor 


\section{Introduction}

In fields such as the structural health monitoring of large-scale civil engineering projects, and seismic wave detection of oil and gas field exploration, the requirements for automated measurement and high-precision testing are becoming increasingly higher, proposing more stringent standards for the accuracy, reliability, stability, and other indictors of vibration sensors; and the sensors must pass tests on their quality and performance to ensure engineering safety and economic feasibility [1-3]. As a result, developing novel low-frequency vibration sensing technologies such as the acceleration sensors is of very important significance [4,5], and the emergence of optical fiber sensing technology has shed a new light on the vibration measurement of acceleration sensors. At present, a variety of new-type FBG acceleration sensors have been developed at home and abroad. Compared with traditional acceleration sensors, they are not only resistant to electromagnetic interference, but also small in size, light in weight, wide in dynamic range, high in accuracy, and can work in harsh environments, therefore they have received wide attention from developed countries [6-8].

In recent years, domestic and foreign scholars have carried out extensive researches on FBG acceleration sensors [9]. For example, Weng Yinyan et al. [10] developed a type of 3D FBG acceleration sensor based on capillary steel tube structure, which has good performance in frequency detection and lateral anti-interference, but its sensitivity is relatively low. Om Prakash et al. [11] proposed a novel mechanical sensor structure based on T-shaped beams, they integrated two fiber gratings in a differential sensing device to improve the sensitivity of the sensor. Camilo A. R. Dia [12] designed a low-cost dynamic-measurement FBG sensor, which used an optical accelerometer and an impulse wave probe to achieve dynamic FBG measurement as high as up to $5 \mathrm{kHz}$. Wen Wang et al. [13] proposed a high-sensitivity FBG acceleration sensor using fibers with suspended micro cores, with a closed cavity structure composed of suspended-core fibers embedded with hollow core fibers, it achieved high-sensitive and stable response to low-frequency signals. However, due to its inherent simultaneous sensitivity to temperature and strain, fiber gratings are susceptible to temperature interference during vibration measurement in actual engineering projects and 
energy applications. In recent years, although FBG acceleration sensors have achieved a series of fruitful results, the problem of cross-sensitivity of temperature and strain has always been a bottleneck hindering the application of FBG acceleration sensing technology.

As a result, targeting at the above-mentioned problems, this study proposed a new-type temperature-insensitive acceleration sensor with sinusoid-shaped curved beams. With fiber gratings pasted on the gradient strain areas of the beams, the applied tensile load made the fiber gratings produced a chirp effect; then the acceleration sensitivity and resonant frequency of the sensor were subject to theoretical analysis and the structural parameters were optimized; after that, the finite element software was adopted to conduct simulation analysis on the structural strain and modal characteristics of the sensor, and a sensor prototype was fabricated to test the performance of the proposed sensor.

\section{Sensor design}

\subsection{Sensor structure}

The proposed sinusoid-shaped double-beam FBG acceleration sensor is composed of a mass block, two sinusoid-shaped beams, a base, and fiber gratings, as shown in Figure 1, the sinusoid-shaped beams are the main elastic components of the structure, they are composed of two symmetrical sinusoid-shaped beams with gradient curvature. Compared with single beam structure, the double-beam structure has stronger torsion resistance. When fabricating the proposed sensor, the phase mask technology was applied to write Bragg gratings into the optical fiber, and a full-adhesive packaging method was adopted as the assembly method, that is, the fiber gratings were in direct contact with the sensor structure, making the fiber gratings more likely to produce the chirp effect. As for the fixation of the fiber gratings, a heavy-weight method was applied, that is, one end of the fiber was fixed to the left side plate by a pressure block, for the other end of the fiber, a weight of $2 \mathrm{~g}$ was applied to prestress the fiber gratings, which were glued to the sinusoid-shaped curved beams with UV glue, and the angle between its axial direction and the neutral plane of the beam was marked as $\theta$. To achieve maximum sensor sensitivity, the oblique angle was set to $\theta=45^{\circ}$. 
(a)

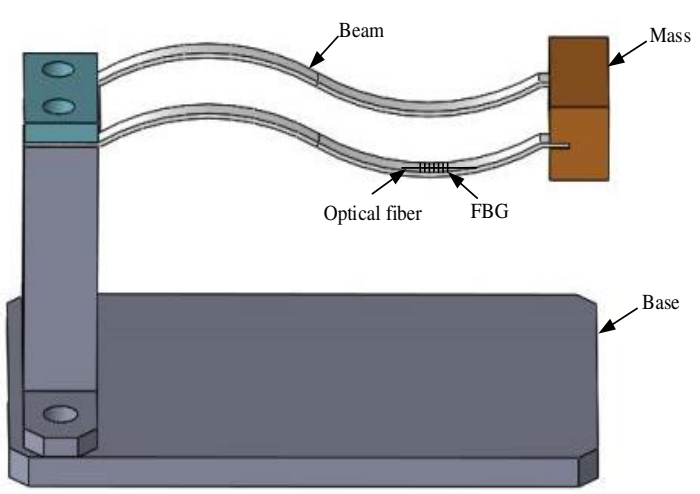

(b)

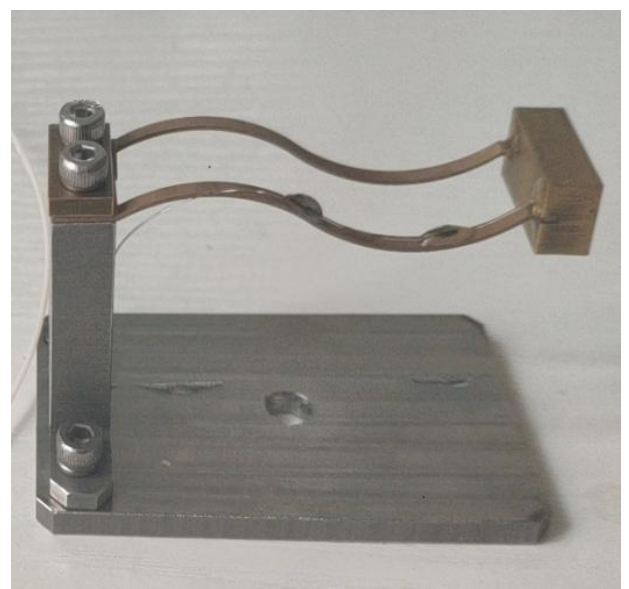

Figure 1 Images of the proposed sensor (a) a diagram of the sensor structure (b) a photo of the

\section{prototype sensor}

When the sensor is excited by an external vibration signal, the mass block at its free end will vibrate with the inertial force, and the vibration will produce strain on the beams. Then, as the beams are bent, the strain generated in different thickness layers will present a gradient distribution, and the strain will be passed on to the fiber gratings, thereby causing the chirp effect; since the impact of temperature on various parts of the plastic beams and the fiber gratings is the same, under the premise that the temperature of the entire elastic beams is the same, the temperature change can only cause the reflection wavelength of each part of the fiber grating to drift together, that is, it only affects the central reflection wavelength of the fiber gratings, and has no effect on the bandwidth. Therefore, such curvature sensing method is not sensitive to temperature.

\subsection{Theoretical analysis of the sensor}

The key to realize temperature-insensitivity of the sensor is to make the fiber gratings produce the chirp effect, as shown in Figure 2, the FBGs were obliquely pasted on the surface of the sinusoid-shaped beams, when the beams are bent, the reflection spectrum bandwidth of the FBGs was broadened under the action of the gradient strain, thereby causing the chirp effect, and the greater the chirp effect of the fiber gratings, the wider the reflection spectrum bandwidth. 


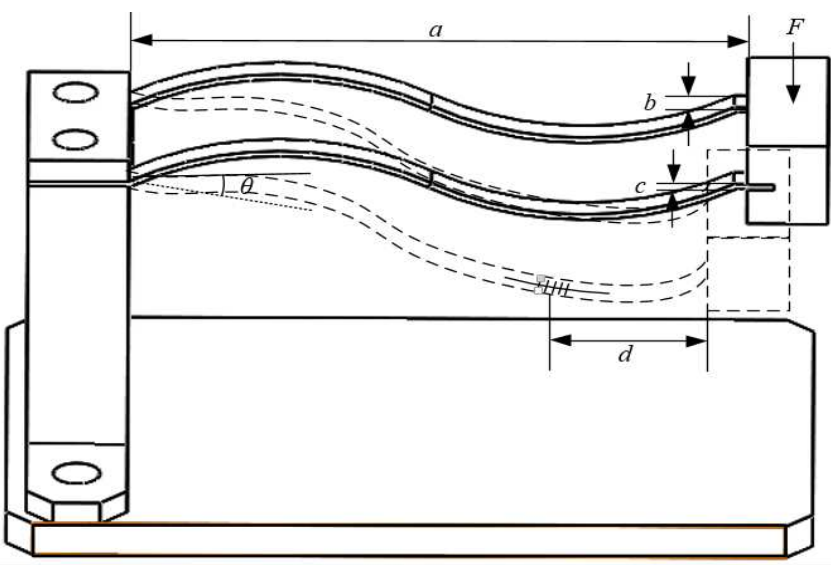

Figure 2 Model of FBG sensing mechanics

To facilitate the description of the beam layers of different thicknesses, the $\mathrm{z}$-axis was defined to be the coordinate axis perpendicular to the neutral plane and with the neutral plane as the zero point, $\mathrm{z}$ is the distance between any point on the grating and the neutral plane, $z \in\left[-z_{0} / 2, z_{0} / 2\right], \mathrm{z}_{0}$ is the initial length of the grating.

According to material mechanics, the axial strain $\varepsilon(z)$ of the grating region can be expressed as:

$$
\varepsilon(z)=\kappa \mu \cos \theta=\kappa \mu \frac{6 F d \sin (2 \theta) z}{E a^{3} b c}
$$

Where, $\kappa$ is the curvature of the beam, when $\kappa$ is constant, the strain along the axis of the fiber grating presents obvious gradient distribution; $\mu$ is the Poisson's ratio; $a$ is the length of the beam; $b$ is the thickness of the beam; $c$ is the width of the beam; $d$ is the distance between the stress point and the midpoint of the fiber grating; $E$ is the Young's modulus of the beam.

For fiber gratings with a uniformly distributed initial grating pitch, under the action of a non-uniform environmental field, the reflection spectrum of the gratings will produce the chirp effect, and the change of FBG wavelength difference caused under the action of the axial non-uniform strain field is:

$$
d \lambda=\kappa \mu \lambda_{0}\left(1-p_{e}\right) \frac{6 F d}{E a^{3} b c} \sin (2 \theta) d z
$$


Where, $p_{e}$ is the effective elastic-optical coefficient of the fiber, which is about $0.22 ; \lambda_{0}$ is the initial center wavelength of the fiber gratings.

The reflection spectrum bandwidth of the entire FBG is the sum of the wavelength changes of each small FBG sections, and the broadening amount of the reflection spectrum bandwidth of the gratings can be expressed as:

$$
\Delta \lambda_{\text {chirp }}=\int_{-z_{0} / 2}^{z_{0} / 2} d \lambda=\frac{6 \kappa \mu\left(1-p_{e}\right) d \sin (2 \theta) z_{0}}{E a^{3} b c} F
$$

With the broadening of the grating reflection spectrum, the intensity of the reflected light will increase accordingly. The relationship between the grating reflected light intensity increment $\Delta P$ and the corresponding bandwidth change $\Delta \lambda_{B W}$ is:

$$
\Delta P=k \alpha^{2} R P_{B B S}(\lambda) \cdot \Delta \lambda_{\text {chirp }}
$$

Where, $k$ and $\alpha$ are respectively the light intensity coupling coefficient and the fiber bending loss of the device connection; $R$ and $\Delta \lambda_{B W}$ are respectively the variations of the grating reflectivity and reflection spectrum bandwidth; $P_{B B S}(\lambda)$ is the output spectrum density of the bandwidth light source, and for light source with flat gain, there is $P_{B B S}(\lambda)=P_{B B S}$

It can be seen from Formulas (3) and (4) that the grating reflection spectrum bandwidth and the reflected light intensity both have a linear relationship with the acceleration change, and have no relationship with the temperature changes. Therefore, the bandwidth tuning technology can eliminate the temperature cross-sensitivity effects, thereby gaining automatic temperature compensation.

\subsection{Sensor quality factor}

The two most important technical parameters of acceleration sensors are the acceleration sensitivity $S$ and the resonant frequency $f_{0}$, and the two are mutually restrictive. The increase in sensitivity will inevitably be accompanied by a decrease in the resonant frequency, and vice versa $[14,15]$. Therefore, the product of resonant frequency and sensitivity had been introduced as a quality factor of the sensor, providing a possibility for the comparison of the 
comprehensive performance of acceleration sensors with different structures, instead of only pursuing high sensitivity or low resonant frequency one-sidedly.

The resonant frequency of the FBG acceleration sensor determines the input signal frequency that can be measured. In fields such as health monitoring of large-scale structures, and early-warning of earthquakes and tsunamis, small-amplitude vibration signals with a frequency below $100 \mathrm{~Hz}$ are often very important. According to dynamic equations, the resonant frequency $f_{0}$ of the entire system can be obtained as:

$$
f_{0}=\frac{1}{2 \pi} w=\frac{1}{2 \pi} \kappa \mu a b \sqrt{\frac{E a b c}{12 m}}
$$

The sensitivity of the FBG acceleration sensor can be understood as the expression of the minimum seismic signal that can be distinguished. In order to obtain low-frequency vibration signals, the sensor needs to have good sensitivity within a certain frequency band. Similar to the principle of the mechanical model shown in Figure 2, when the sensor moves with the object to be measured, its acceleration $a$ produces an opposite inertial force $F$ on the mass block, then the mass block is forced to vibrate, causing the center of the S-shaped beam to shift.

According to the definition of the sensitivity of acceleration sensor, combining with Formula (3), the sensor acceleration sensitivity $\mathrm{S}$ can be expressed as:

$$
S=\frac{\Delta \lambda_{\text {chir }}}{a}=\frac{6 \kappa \mu\left(1-p_{e}\right) d \sin (2 \theta) l_{0} m}{E a^{3} b c}
$$

Where, $m$ is the mass of the mass block; and the sensitivity referred to in this paper is the peak-to-peak sensitivity $2 S$.

\section{Structural parameter optimization}

According to the theoretical analysis results of Formulas (5) and (6), the length $a$, width $b$, thickness $c$ and mass $m$ of the sinusoidal-shaped curved beams are the four key parameters affecting the sensitivity and resonant frequency of the FBG acceleration sensor. For the measurement of low-frequency and weak vibration signals, it is necessary to optimize the design of the sensor to increase its sensitivity as much as possible, and assign a suitable value to the resonant frequency to ensure that the sensor could achieve high-sensitivity indicators within a good frequency response range. By comprehensively considering the 
performance requirements, manufacturing process, mechanical size, the materials and other factors of the sensor, according to Formulas (5) and (6), under the condition that other parameters were given, the impact of parameters that can be flexibly adjusted in the sensor structure on the sensitivity and resonant frequency of the sensor had been taken into consideration to determine a reasonable optimization scheme. During sensor fabrication, beryllium bronze was chosen as the material of the beams, the Young's modulus $E=105.14$ $\mathrm{GPa}$, the Poisson's ratio $\mu=0.28$, the initial center wavelength of the fiber grating $\lambda_{0}=1549.5$ $\mathrm{nm}$, and the initial length of the grating $z_{0}=5 \mathrm{~mm}$.

First of all, since the axial size of the sensor should be as small as possible to facilitate actual installation and use, the width of the sensor beam was set to $b=2 \mathrm{~mm}$, and the thickness was $c=1 \mathrm{~mm}$, combining with Formulas (5) and (6), the impact of the length of the S-Shaped curved beams on the sensitivity and resonant frequency was examined, and Figure 3 shows the changes of sensitivity $\boldsymbol{S}$ and resonant frequency $f_{0}$ with $\boldsymbol{a}$, it can be seen from the figure that, when $\boldsymbol{a}$ increases, $\boldsymbol{S}$ decreases accordingly, and $f_{0}$ increases rapidly, and when $\boldsymbol{a}$ is greater than $40 \mathrm{~mm}$, the change tends to be stable. To meet the requirements of low-frequency vibration signal measurement for higher sensitivity and smaller resonant frequency, the value of $\boldsymbol{a}$ could be taken as greater than $40 \mathrm{~mm}$ to meet the requirements of the overall structural size of the sensor.

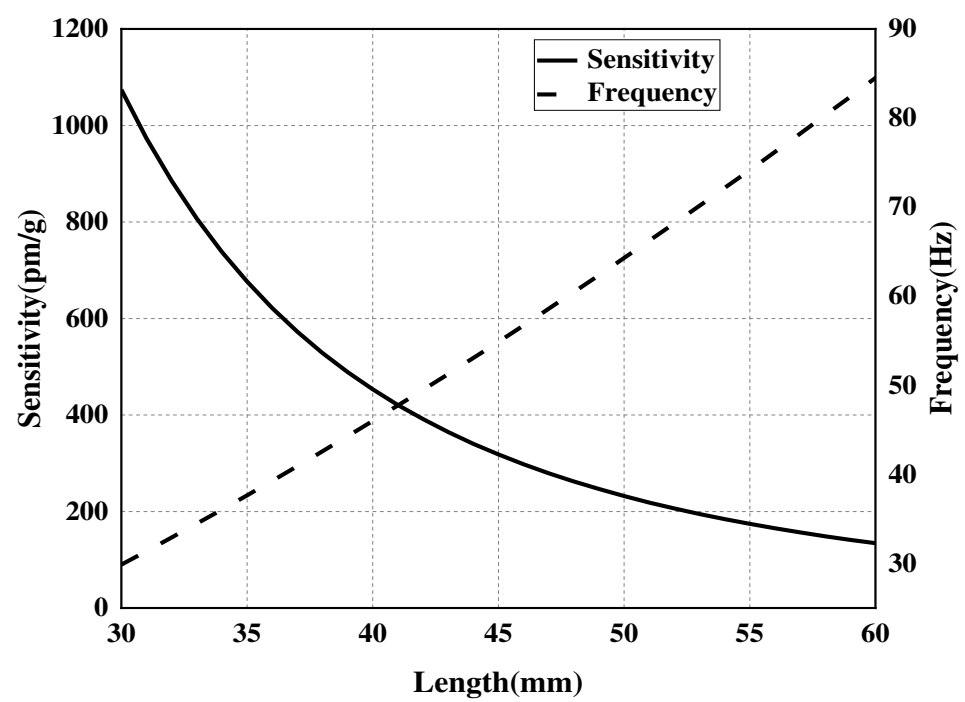


In view of the constraints of the assembly structure and size of the sensor, the width of the beams was adjusted. The length of the beams was set to $a=45 \mathrm{~mm}$ and the thickness was set to $c=1 \mathrm{~mm}$, combining with Formulas (5) and (6), the impact of the width of the S-shaped curved beams on the sensitivity and resonant frequency of the sensor was investigated, and Figure 4 shows the changes of sensitivity $\boldsymbol{S}$ and resonant frequency $f_{0}$ with $\boldsymbol{b}$, it can be seen from the figure, with the increase of width $\boldsymbol{b}$, the sensitivity $\boldsymbol{S}$ decreases rapidly, and the resonant frequency $f_{0}$ increases, when width $b$ was between $1 \mathrm{~mm}$ and $3 \mathrm{~mm}$, the sensitivity and resonant frequency reached the requirements of low frequency vibration measurement.

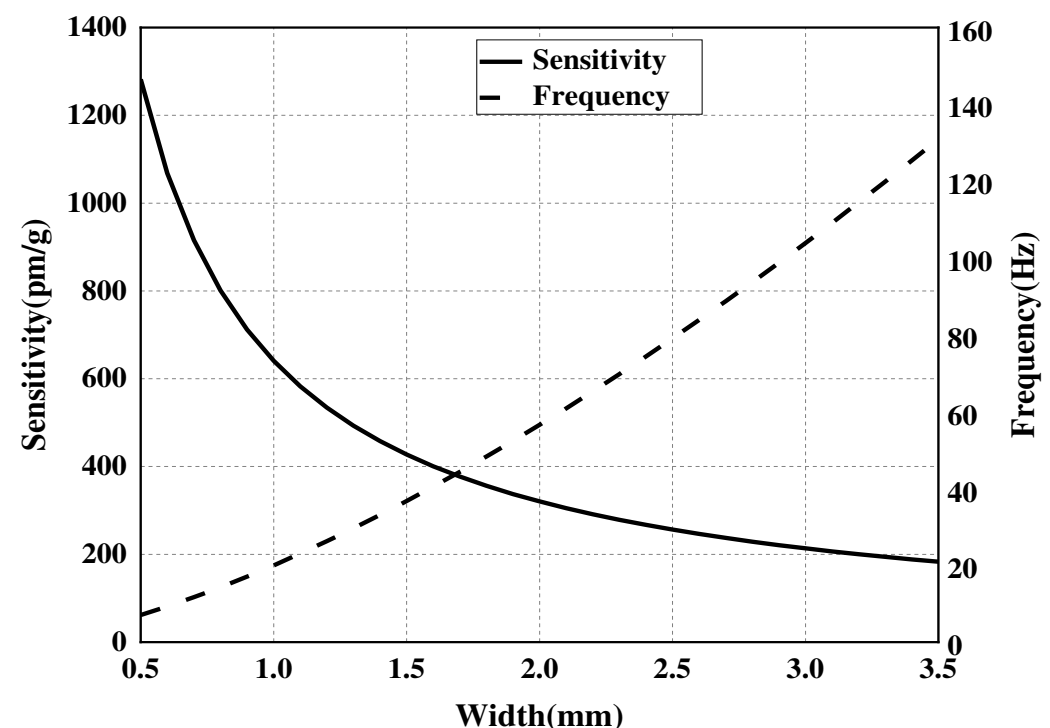

Figure 4 Changes of sensitivity $\mathbf{S}$ and resonant frequency $f_{0}$ with width $\mathbf{b}$

The length of the sensor beams was taken as $a=45 \mathrm{~mm}$, and the width $b=2 \mathrm{~mm}$, combining with Formulas (5) and (6), the impact of the thickness $c$ of the S-shaped curved beams on the sensitivity and resonant frequency of the sensor was investigated, and Figure 5 shows the changes of sensitivity $\boldsymbol{S}$ and resonant frequency $f_{0}$ with $\boldsymbol{c}$, it can be seen from the figure, with the increase of thickness $\boldsymbol{c}, \boldsymbol{S}$ decreases rapidly, and $f_{0}$ increases accordingly, 210 therefore, the beams should be as thin as possible. Considering the actual materials and 211 processing conditions, the sensor with a beam thickness of $0.5 \mathrm{~mm}$ could be chosen for the 212 tests. 


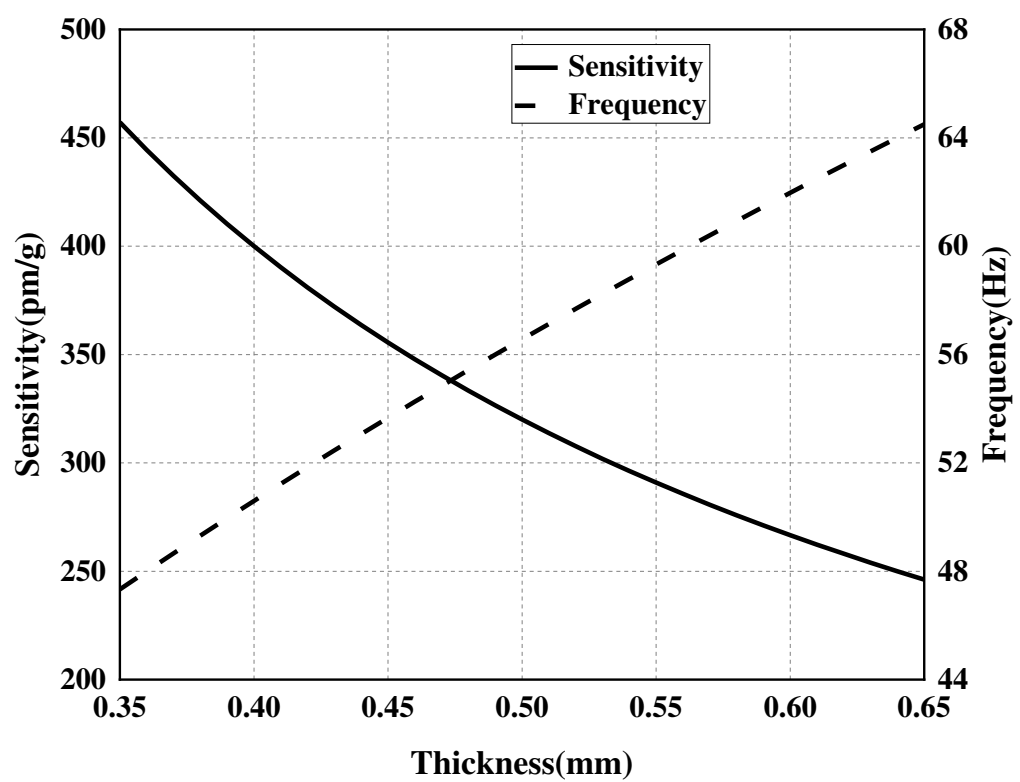

Figure 5 Changes of sensitivity $\mathbf{S}$ and resonant frequency $f_{0}$ with thickness c

At last, the impact of the mass of the mass block $\boldsymbol{m}$ was investigated, as shown in Figure

6, increasing mass $\boldsymbol{m}$ would increase sensitivity $\boldsymbol{S}$ and decrease resonant frequency $f_{0}$.

217 However, considering the size factor, the mass block cannot be made too big, so the brass

218 with a relatively large density had been chosen as the material of the mass block, and the mass of the mass block was calculated to be about $0.007 \mathrm{~kg}$.

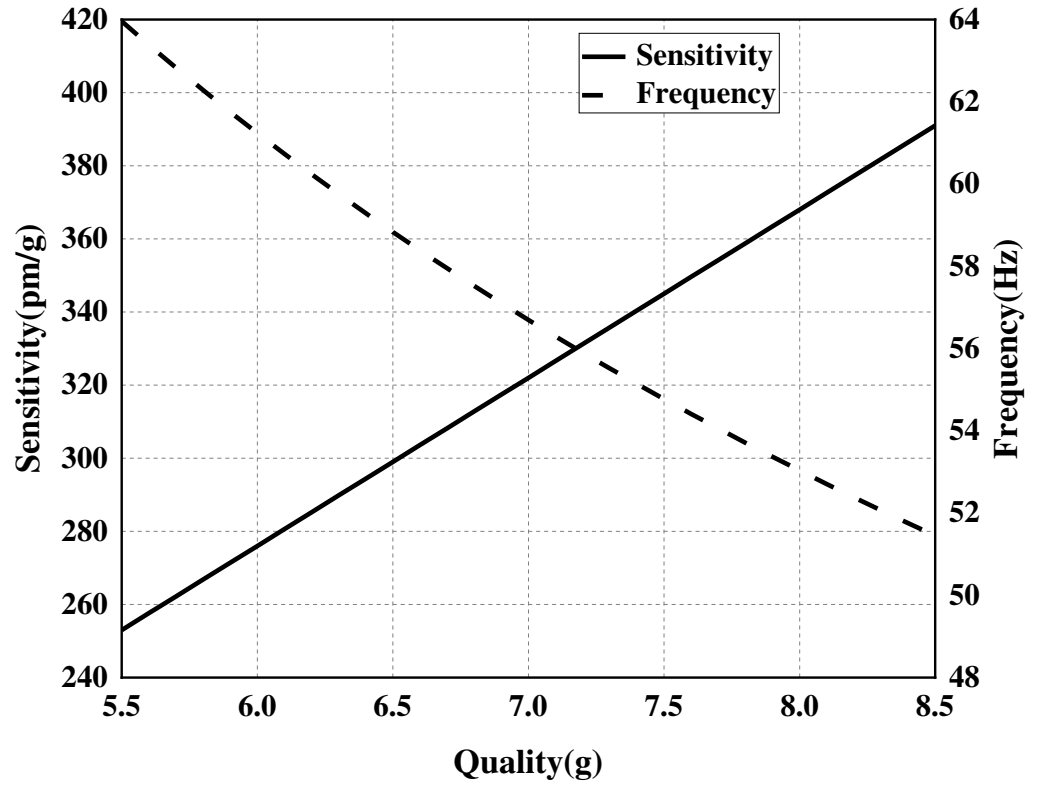


The above analysis has given the impact of the four key parameters on the sensitivity and resonant frequency of the sensor. In summary, the structural parameters and materials of 224 the acceleration sensor were set as shown in Table 1.

Table 1 Structural parameters of the FBG acceleration sensor

\begin{tabular}{cccc}
\hline Structural parameters & Name & Value & Material \\
\hline$a$ & Length of the beam & $45 \mathrm{~mm}$ & Beryllium \\
$b$ & Width of the beam & $2 \mathrm{~mm}$ & bronze \\
$c$ & Thickness of the beam & $0.5 \mathrm{~mm}$ & \\
$m$ & Mass of the mass block & $0.007 \mathrm{~kg}$ & Brass \\
\hline
\end{tabular}

\section{3. Finite element simulation}

\subsection{Strain analysis of sensor structure}

The ANSYS software was adopted for finite element simulation analysis. First, relevant constraints were established and the left end of the beams was fixed, the connection surface of the beams and the mass block was set to be a fully bound support constraint, and a concentrated load with a size of the earth's gravitational acceleration $g\left(g=9.8 \mathrm{~m} / \mathrm{s}^{2}\right)$ was applied to the free end of the beams, other parameters of the materials of the model were also set as shown in Table 2.

Table 2 Parameters of model materials

\begin{tabular}{cccccc}
\hline Part & Material & Category & $\begin{array}{c}\text { Elastic } \\
\text { Modulus }(\mathrm{Pa})\end{array}$ & $\begin{array}{c}\text { Poisson's } \\
\text { ratio }\end{array}$ & $\begin{array}{c}\text { Density } \\
\left(\mathrm{Kg} / \mathrm{m}^{3}\right)\end{array}$ \\
\hline Shell & Structural Steel & Structural Steel & $2.0 \times 10^{11}$ & 0.30 & 7850 \\
Beams & Beryllium bronze & Beryllium & $1.05 \times 10^{11}$ & 0.30 & 8300 \\
Mass block & Brass alloy & Brass & $1.0 \times 10^{11}$ & 0.35 & 7600 \\
\hline
\end{tabular}

As shown in Figure 7, the model was meshed for static stress simulation analysis, it can be seen from the figure, the strain on different layers of the beams presented a gradient distribution, the maximum deformation at the free end was $0.396 \mathrm{~mm}$, when the beams were bent, the grating areas deformed. 


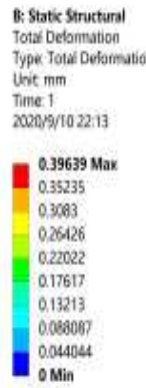

(a)

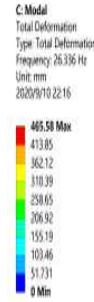

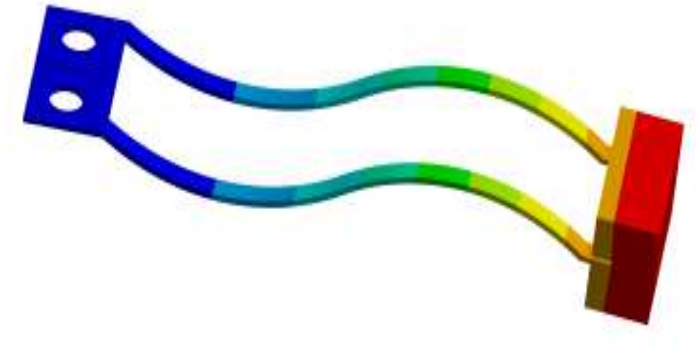
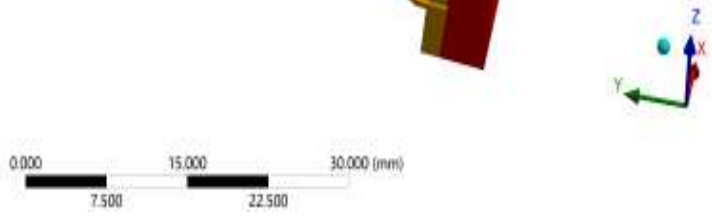

Figure 7 Static stress simulation analysis

\subsection{Modal analysis of sensor structure}

Modal is an inherent property of the sensor structure. According to the static stress analysis results of the sensor, the sensor prototype was subject to modal analysis. The calculation order was set to 4 , and the first-order, second-order, third-order, and fourth-order modal frequency was $53 \mathrm{~Hz}, 119 \mathrm{~Hz}, 503 \mathrm{~Hz}$, and $683 \mathrm{~Hz}$, respectively. The first-order and second-order modal analysis diagrams are shown as Figure 8 below.
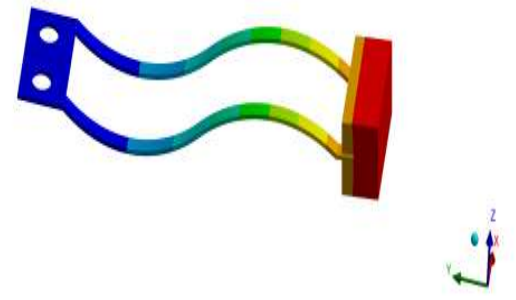

(b)

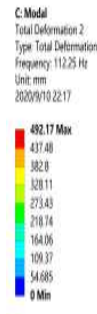

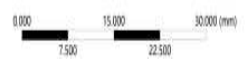
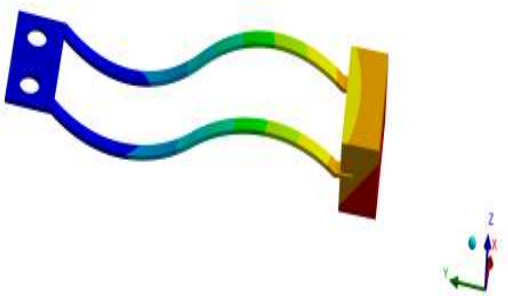

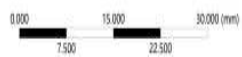

Figure 8 Modal analysis diagrams (a) First-order mode (b) Second-order mode

As shown in Figure 8, the first-order mode is the working vibration mode, indicating that the model vibrated along the Y-axis under the action of external vibration, and the first-order mode had a frequency of about $53 \mathrm{~Hz}$; the second-order mode is the torsional vibration mode, indicating that the model twisted around the $\mathrm{X}$ axis under the action of external vibration. Comparing the modal frequencies of each order, it can be seen that the 
255 first-order modal frequency was quite different from the second, third, and fourth-order 256 modal frequencies, indicating that the structure had a smaller cross-coupling.

\section{4. Test on the proposed sensor}

The sensor test system consisted of a broadband light source, a coupler, a vibration table system, an optical power meter, a spectrum analyzer and a computer, as shown in Figure 9. Wherein the signal function generator of the vibration table system was the RIGOL series DG1022 model produced by RIGOL Technologies, its sampling rate is $1 \mathrm{GSa} / \mathrm{a}$, with 14 kinds of quasi-waveform functions and standard configuration interfaces, and it supports users to remotely control the instrument and USB interface data transmission; the signal amplifier adopted the MYW-TZQ50 model produced by Beijing Weiyun Company, its frequency response range is $1-15000 \mathrm{~Hz}$, the signal-to-noise ratio is greater than $75 \mathrm{~dB}$, together with the signal function generator, they can amplify the function signals. In the test, the light wave of the broadband light source was delivered to the acceleration sensor on the vibration table system, the reflected light of the fiber gratings entered the spectrum analyzer or the optical power meter through a same fiber coupler, then the reflection spectrum bandwidth of the sensor was measured by the spectrum analyzer, and the power of the reflected light of the sensor was measured by the optical power meter, moreover, combining with the grating chirp method, the measurement of the acceleration was realized, the amplitude-frequency response characteristics, linear response characteristics, and temperature performance of the sensor were studied, and the data was subject to normalization processing to obtain the performance parameters of the sensor. 


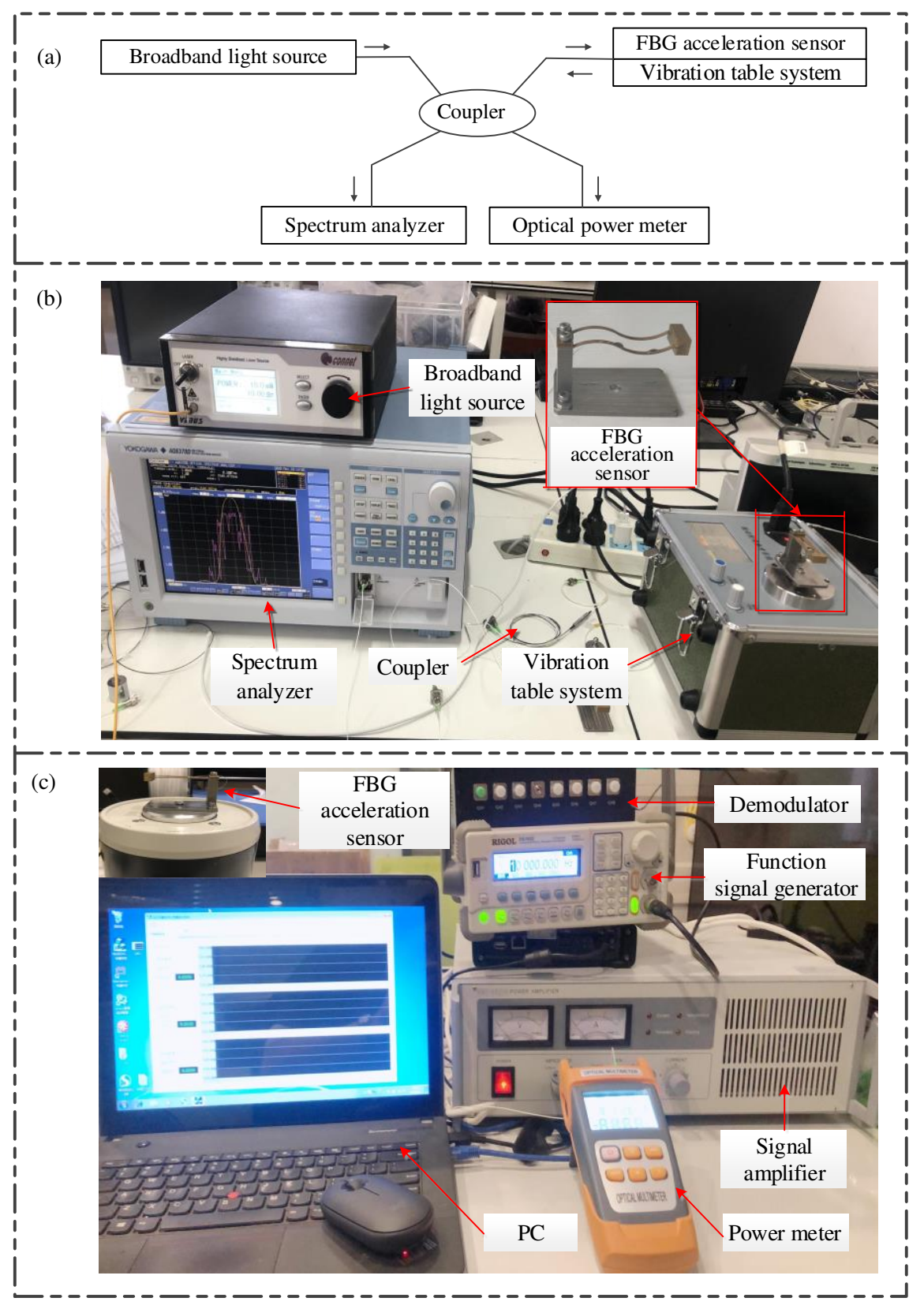

Figure 9 Sensor test system (a) A diagram of the test system (b) Measurement of reflection spectrum bandwidth (c) Measurement of reflected light power

\subsection{Test on sensor reflection spectrum}

In order to verify whether the sensor had produced a chirp effect, the AQ63700 model high-precision spectrum analyzer produced by Japanese company Yokogawa was adopted to test the reflection spectrum of the proposed sensor. The proposed sinusoid-shaped curved beam sensor was fixed on a model JX-3 vibration calibrator produced by Beijing Weiyun Company. The vibration calibrator can display the three ranges of displacement, velocity and acceleration at the same time, and it can switch among 9 frequencies between 5-995 Hz. The 
center wavelength of the FBG was $1549.5 \mathrm{~nm}$, the reflectivity was 0.999 , and the initial bandwidth was $0.2 \mathrm{~nm}$. The temperature of the grating area was constant at $25^{\circ} \mathrm{C}$. On the vibration calibrator, the frequency was fixed at $40 \mathrm{~Hz}$, and the value of acceleration was changed from $0 \mathrm{~g}$ to $4 \mathrm{~g}$ in $0.2 \mathrm{~g}$ steps. The changes in the reflection spectrum bandwidth of the sinusoid-shaped curved beam sensor were observed, then, under the same test conditions, the test was repeated on an ordinary rectangular-shaped beam sensor fixed on the vibration calibrator, as shown in Figure 10, (a) is the reflection spectrum bandwidth of chirp effect produced by the sinusoid-shaped curved beam sensor under different accelerations, and (b) is the reflection spectrum bandwidth of chirp effect produced by the rectangular-shaped beam sensor under different accelerations.

(a)

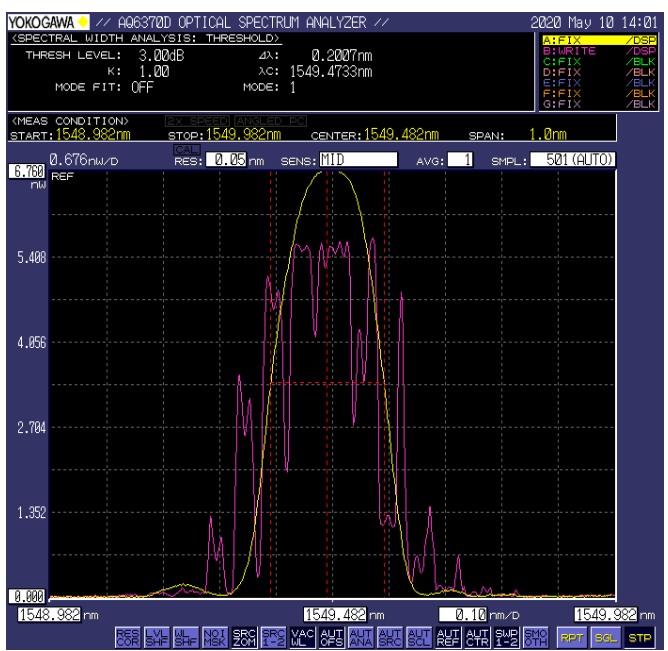

(b)

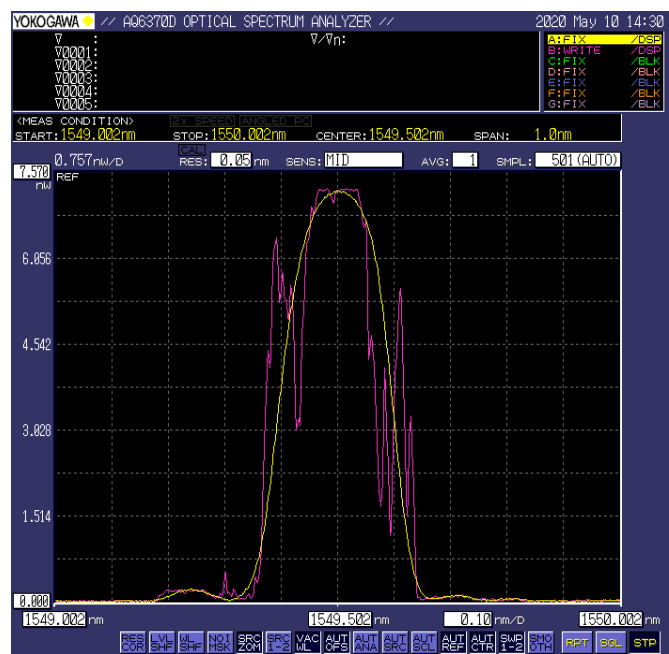

Figure 10 Reflection spectra under different accelerations: (a) The reflection spectrum bandwidth of the chirp effect produced by the sinusoid-shaped curved beam sensor under the acceleration of about $1 \mathrm{~g}$; (b) The reflection spectrum bandwidth of the chirp effect produced by the rectangular-shaped beam sensor under the acceleration of about $2 \mathrm{~g}$

In Figure 10, the yellow waveforms are the initial reflection spectrum bandwidth waveforms of the sensor. Then, with the change of acceleration value, the reflection spectra of the two sensors showed obvious deformation and multi-peak phenomenon, that is, the uniform period FBG had produced a chirp effect on the sinusoid-shaped curved beam sensor. The difference is that, when the acceleration value of the sinusoid-shaped curved beam sensor was about 1g, it exhibited a chirp effect with splitting peaks; while for the rectangular-shaped beam sensor, the produced chirp effect was obvious only when the acceleration value reached 
about $2 \mathrm{~g}$. According to comparison and analysis, the sinusoid-shaped curved beam sensor is more prone to produce the chirp effect.

\subsection{Test on the acceleration sensitivity amplitude-frequency response}

\section{characteristics of the sensor}

The test adopted the model MWY-JZQ50 vibration table system produced by Beijing Weiyun Company, its maximum amplitude is $12.5 \mathrm{~mm}$, and the maximum acceleration is 45.5 g. In the test, the amplitude of the vibration signal was fixed, and the acceleration sensor was subject to frequency sweep test. The frequency range was from $5 \mathrm{~Hz}$ to $120 \mathrm{~Hz}$ in $5 \mathrm{~Hz}$ steps. The dynamic response coefficient $\mathrm{C}$ corresponding to each frequency was the ratio of the measured bandwidth of the FBG acceleration sensor to the acceleration value of the vibration table. The amplitude-frequency response curve of the FBG acceleration sensor obtained by calibration is shown in Figure 11.

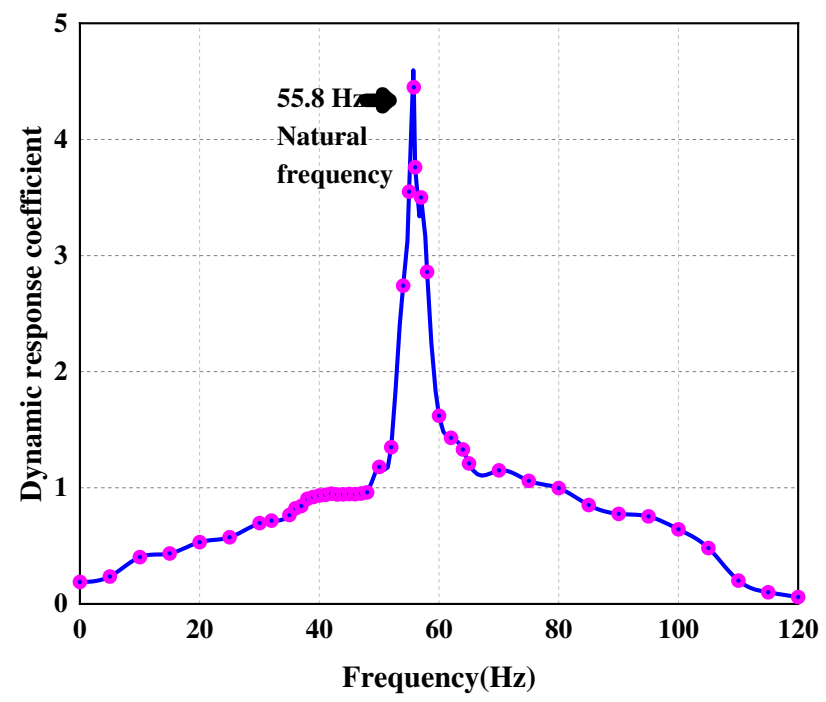

Figure 11 Acceleration sensitivity amplitude-frequency response curve of the acceleration sensor

It can be seen from Figure 11 that when the natural frequency was about $56 \mathrm{~Hz}$, the response was relatively smooth at $30-50 \mathrm{~Hz}$, the result showed that the measured value of the natural frequency was close to the value of $53 \mathrm{~Hz}$ calculated in the previous simulation, and the minor error may be caused by factors such as the loss of the structure during sensor assembly or the poor adjustment of the fiber grating prestress. At the same time, it had met the measurement requirements for low-frequency seismic signals, and the sensor has certain advantages in this frequency response range. Moreover, the sealed package of the sensor as a 
whole had minimized the influence of external environment, thereby ensuring the longest service life of the sensor, so that it can make stable responses to the vibration acceleration signals after long time operations.

\subsection{Test on linear response characteristics}

The linear response of an acceleration sensor refers to the changes in the sensitivity of the sensor with acceleration within a measurable range. In the sensor linear response test, the performance of the sinusoid-shaped curved beam sensor and the ordinary rectangular-shaped beam sensor was compared, the vibration table frequency was set to $40 \mathrm{~Hz}$ to apply a sinusoidal excitation signal to the sensor, and the test range of the acceleration was from $0 \mathrm{~g}$ to $5 \mathrm{~g}$ in $0.2 \mathrm{~g}$ steps. By fitting the linear relationship between the reflection spectrum bandwidth and acceleration, the sensitivity fitting curve of the sensor was obtained, and the slope of the curve represented the acceleration sensitivity of the sensor. In Figure 12, the square dot curve represents the fitting curve of the reflection spectrum bandwidth and acceleration of the sinusoid-shaped curved beam sensor, and the circular dot curve represents the fitting curve of the reflection spectrum bandwidth and acceleration of the ordinary rectangular-shaped beam FBG sensor.

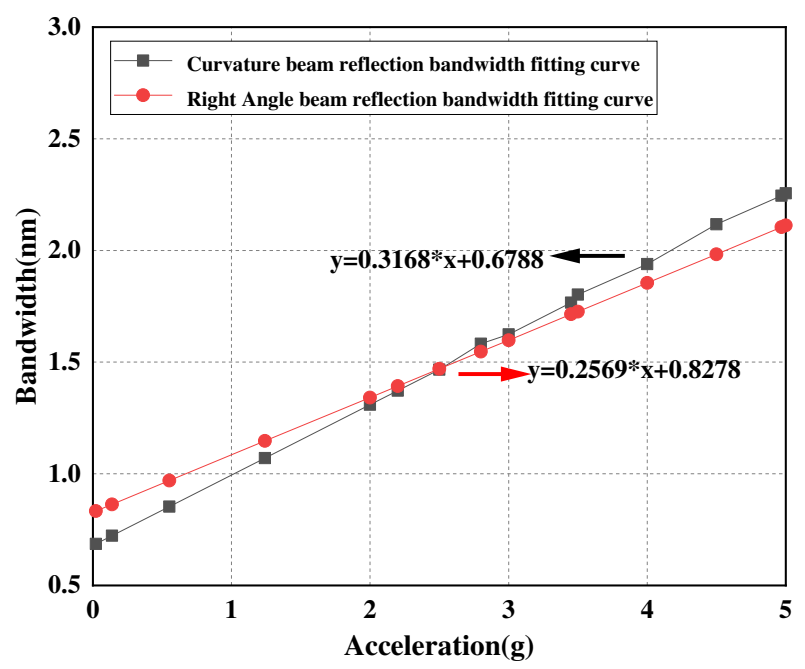

Figure 12 Comparison of reflection spectrum bandwidth fitting curves at two different accelerations

It can be seen from Figure 12 that at a frequency of $40 \mathrm{~Hz}$, the acceleration measurement sensitivity of the sinusoid-shaped curved beam sensor reached $317 \mathrm{pm} / \mathrm{g}$, and the linearity between the reflection spectrum bandwidth, the reflected light power and the acceleration 
was 0.9959 and 0.9992 , respectively. While for the ordinary rectangular-shaped beam FBG sensor, the sensitivity reached $256 \mathrm{pm} / \mathrm{g}$. The results showed that there're good linear relationships between the reflection spectrum bandwidth, the reflected light power and the acceleration, and the acceleration sensitivity of the sinusoid-shaped beam structure had been significantly improved compared with that of the rectangular-shaped beam structure.

\subsection{Test on temperature stability}

A temperature control equipment had been added to the temperature stability test. A temperature control box produced by Zhongke Meiqi Company had been selected for the test; the temperature change program of the equipment can be set in advance, including temperature change amount, change time and hold time, and the temperature control can be accurate to $0.01^{\circ} \mathrm{C}$. Under different accelerations ( $1 \mathrm{~g}, 2 \mathrm{~g}$ and $\left.4 \mathrm{~g}\right)$, the test was conducted using the temperature control box to change the temperature environment of the sensor, the start temperature was set to $5^{\circ} \mathrm{C}$, the end temperature was set to $50^{\circ} \mathrm{C}$, and the change of each temperature node section was $5^{\circ} \mathrm{C}$. When each temperature node was reached, after the temperature had been kept for 2 minutes, the fiber grating reflection spectrum was recorded. Then after the data was normalized, the change of the FBG reflection spectrum bandwidth with temperature was obtained, and a high-resolution AQ63700 spectrum analyzer developed by Yokogawa was used in the test. Then, under different accelerations ( $1 \mathrm{~g}, 2 \mathrm{~g}$ and $4 \mathrm{~g}$ ), the change of FBG reflection spectrum bandwidth with temperature was obtained, as shown in Figure 13. 


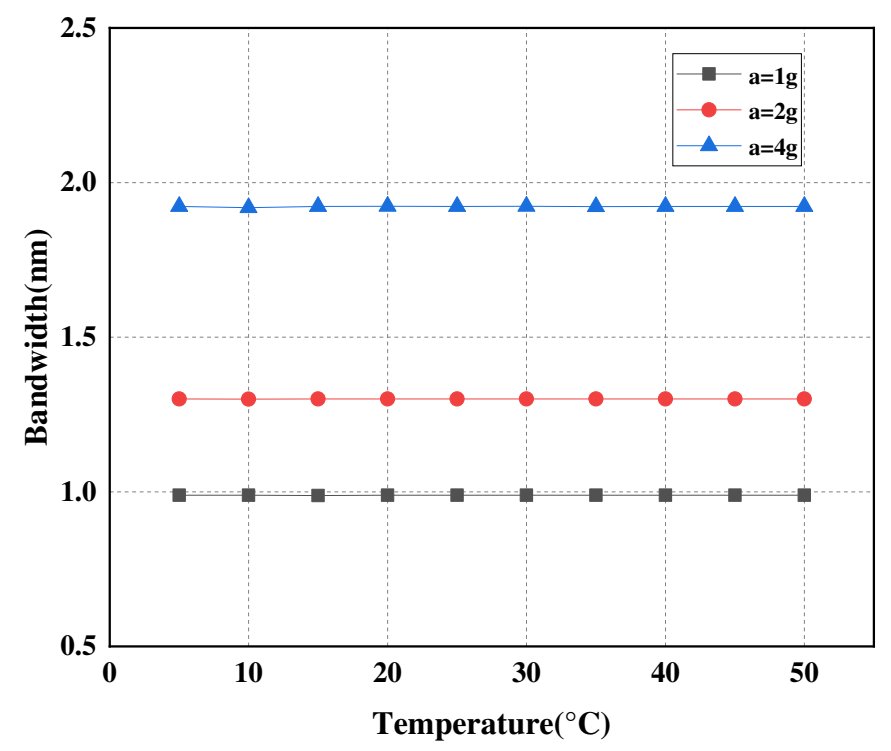

Figure 13 Change of bandwidth with temperature at different accelerations

It can be seen from Figure 13 that when the temperature was increased from $5^{\circ} \mathrm{C}$ to $50^{\circ} \mathrm{C}$, the change in the FBG reflection spectrum bandwidth observed by the spectrometer was always less than $0.05 \mathrm{~nm}$, and the slight fluctuations may be caused by the mismatch between the center of the FBG and the neutral layer of the beams; however, its impact on the test results was tiny and can be ignored, and the results showed that the sensor is not sensitive to temperature changes.

\section{Conclusion}

In this paper, a temperature-insensitive FBG acceleration sensor with sinusoid-shaped curved beams was proposed. In the structure of the sensor, FBGs were uniformed and obliquely pasted to the surface of the beams, when the beams were bent, gradient strain and chirp effect were produced on different layers of the beams, achieving acceleration sensing and temperature self-compensation of the fiber gratings. Then, through theoretical calculation, simulation analysis and test verification, the performance of the proposed sensor and an ordinary rectangular-shaped beam sensor was compared, and the results showed that the proposed sensor had good performance, it's stable, reliable, and insensitive to temperature changes; its sensitivity reached $317 \mathrm{pm} / \mathrm{g}$, which was significantly higher than the ordinary sensor; moreover, the proposed sensor has a natural frequency of about $56 \mathrm{~Hz}$, and it is quite suitable for solving problems such as low-frequency vibration signal collection and 
temperature interference in fields such as building structural health monitoring when seismic signals acting as the excitation source.

\section{Acknowledgments}

This study was financially supported by the National Key Research and Development Programme of China (Grant No 2018YFC1503801), the Fundamental Research Funds for the Central Universities (Grant No ZY20180111), and the Fundamental Research Funds for the Central Universities (Grant No ZY20180241) .

\section{References}

[1] Du C, Dutta S, Kurup P, Yu T, et al. A review of railway infrastructure monitoring using fiber optic sensors.[J]. Sensors \& Actuators A: Physical, 2020, 303.

[2] Wang W, Shen Y, Guo T, et al. Highly sensitive fiber-optic accelerometer using a micro suspended-core fiber.[J]. Applied Optics, 2019, 58(21): 5852-5858.

[3] Wang X.K, Chen J.Y, Chen W.C., et al. Sparse modeling of seismic signals produced by high-speed trains[J]. Chinese Journal of Geophysics, 2019, 62(6): 2336-2343.

[4] Zhou C, Tong X, Mao Y, et al. Study on a high-temperature optical fiber F-P acceleration sensing system based on MEMS[J]. Optics and Lasers in Engineering. 2019, 120: 95-100.

[5] Hong L, Wang J.H, Yao Z.J, et al. Sensitivity improvement of a new structure crack meter with angular adjustment[J]. Measurement and Control. 2019, 52(9-10): 1545-1551.

[6] Li T.L, Tan Y.G, et al. Diaphragm Based Fiber Bragg Grating Acceleration Sensor with Temperature Compensation.[J]. Sensors, 2017, 17(1): 218-224.

[7] Liu Bin, Karanja, Joseph M, et al. Medium-high frequency FBG accelerometer with integrative matrix structure[J]. Applied Optics, 2015, 54(11): 3115-3121.

[8]Wu X , Wang X , Li S, et al. Cantilever Fiber-Optic Accelerometer Based on Modal Interferometer[J]. Photonics Technology Letters, IEEE, 2015, 27(15):1632-1635.

[9] Xueguang Qiao, et al.Temperature-independent refractive index measurement based on Fabry-Perot fiber tip sensor modulated by Fresnel reflection[J].Chinese Optics Letters,2012,10(05):23-26.

[10]Yinyan Weng et al.Compact FBG diaphragm accelerometer based on L-shaped rigid cantilever beam[J].Chinese Optics Letters,2011,9(10):22-25. 
419 [11] Parida O P, Nayak J, et al. Design and Validation of a Novel High Sensitivity 420 Self-Temperature Compensated Fiber Bragg Grating Accelerometer[J]. IEEE Sensors Journal, $421 \quad 2019,19(15):$ 6197-6204.

422 [12] Camilo A, Cátia Leitão, Carlos A, et al. Low-Cost Interrogation Technique for Dynamic 423 Measurements with FBG-Based Devices[J]. Sensors, 2017,17(10):2414-2423.

424 [13] Wang Wen, Shen Yedi, et al. Highly sensitive fiber-optic accelerometer using a micro 425 suspended-core fiber.[J]. Applied optics, 2019,58(21):5852-5858.

426 [14] Zhou C, Tong X, Mao Y, et al. Study on a high-temperature optical fiber F-P 427 acceleration sensing system based on MEMS[J]. Optics and Lasers in Engineering. 2019, 120: $428 \quad 95-100$.

429 [15] K O Hill, Y Fuji, D C Johnson, et al. Photosensitivity in optical fiber waveguides: 430 Application to reflection filter fabrication[J]. Applied Physics Letters, 2018, 32(10):647-649. 
Figures

(a)

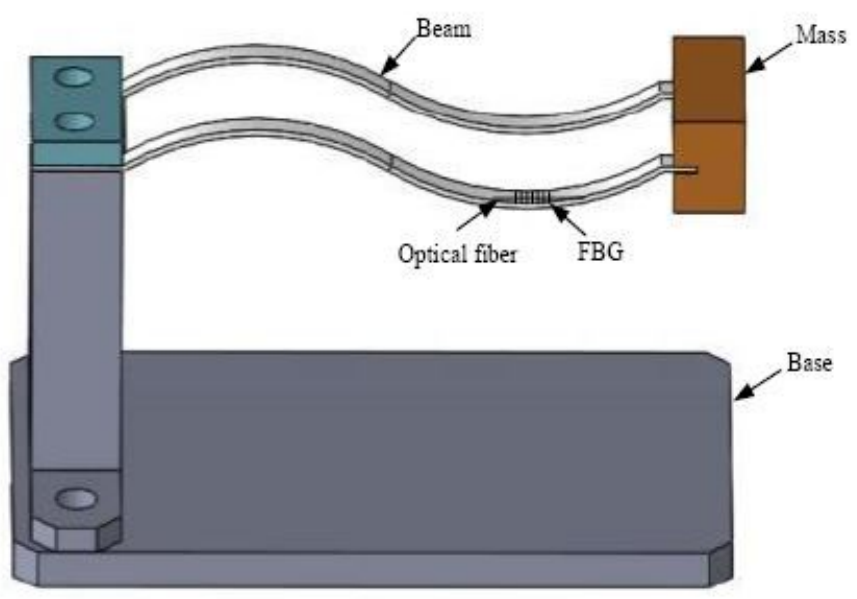

(b)

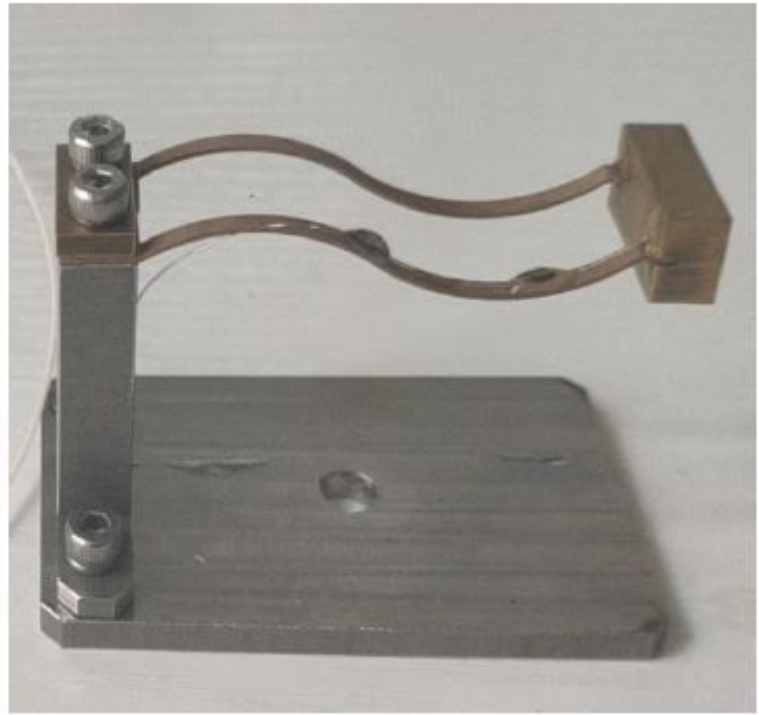

Figure 1

Images of the proposed sensor (a) a diagram of the sensor structure (b) a photo of the prototype sensor

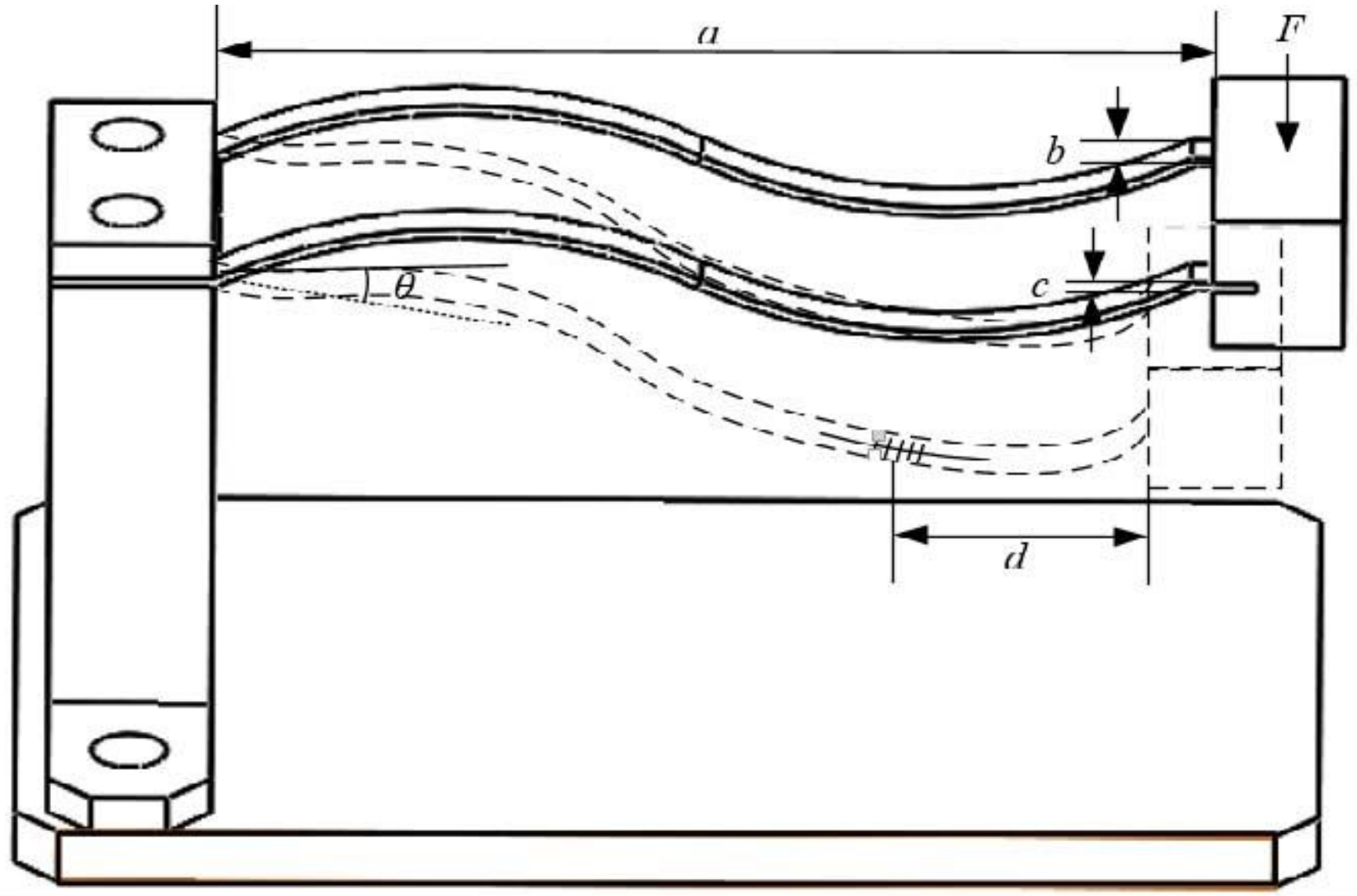

Figure 2 
Model of FBG sensing mechanics

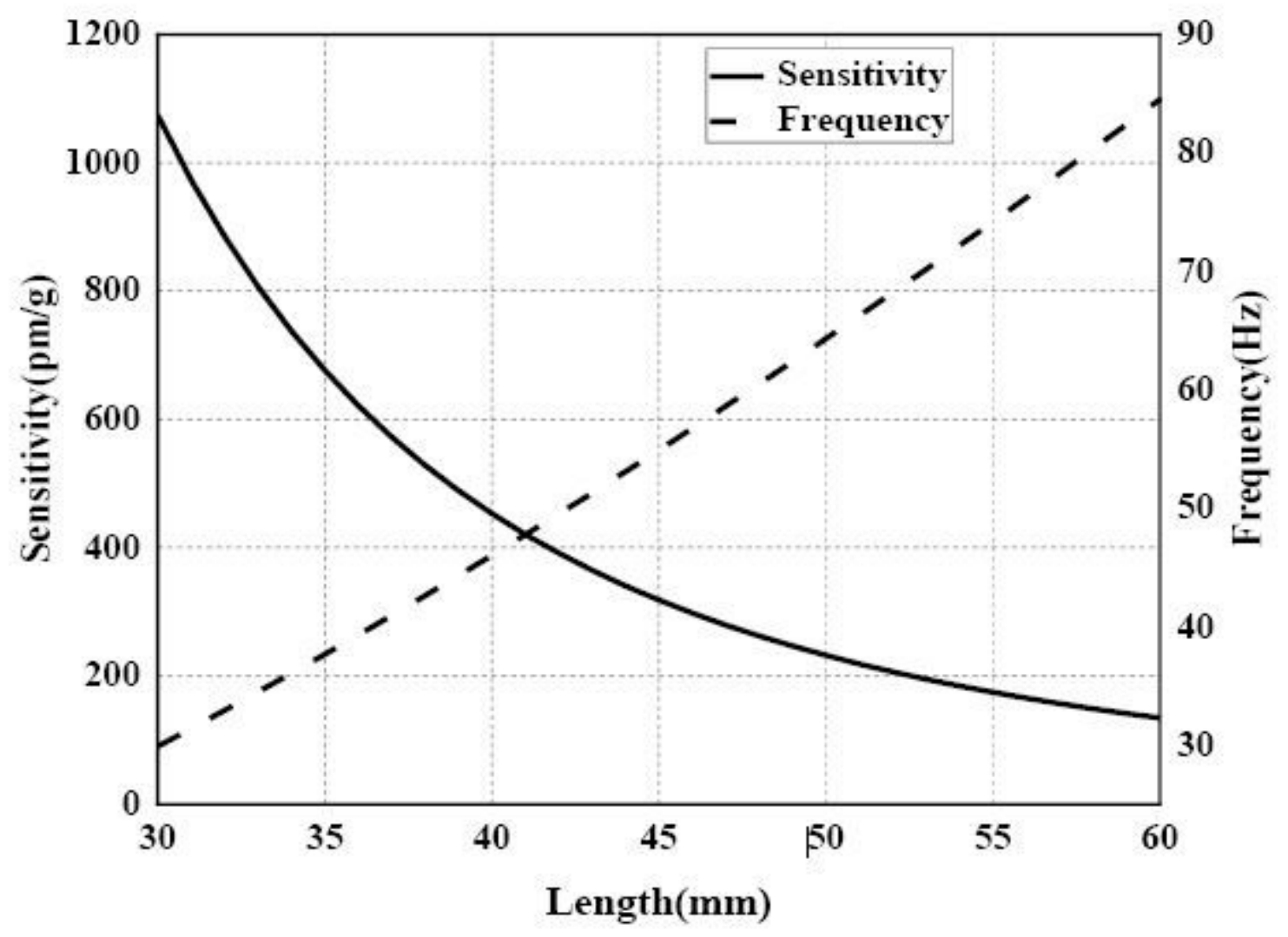

Figure 3

Changes of sensitivity and resonant frequency with length a 


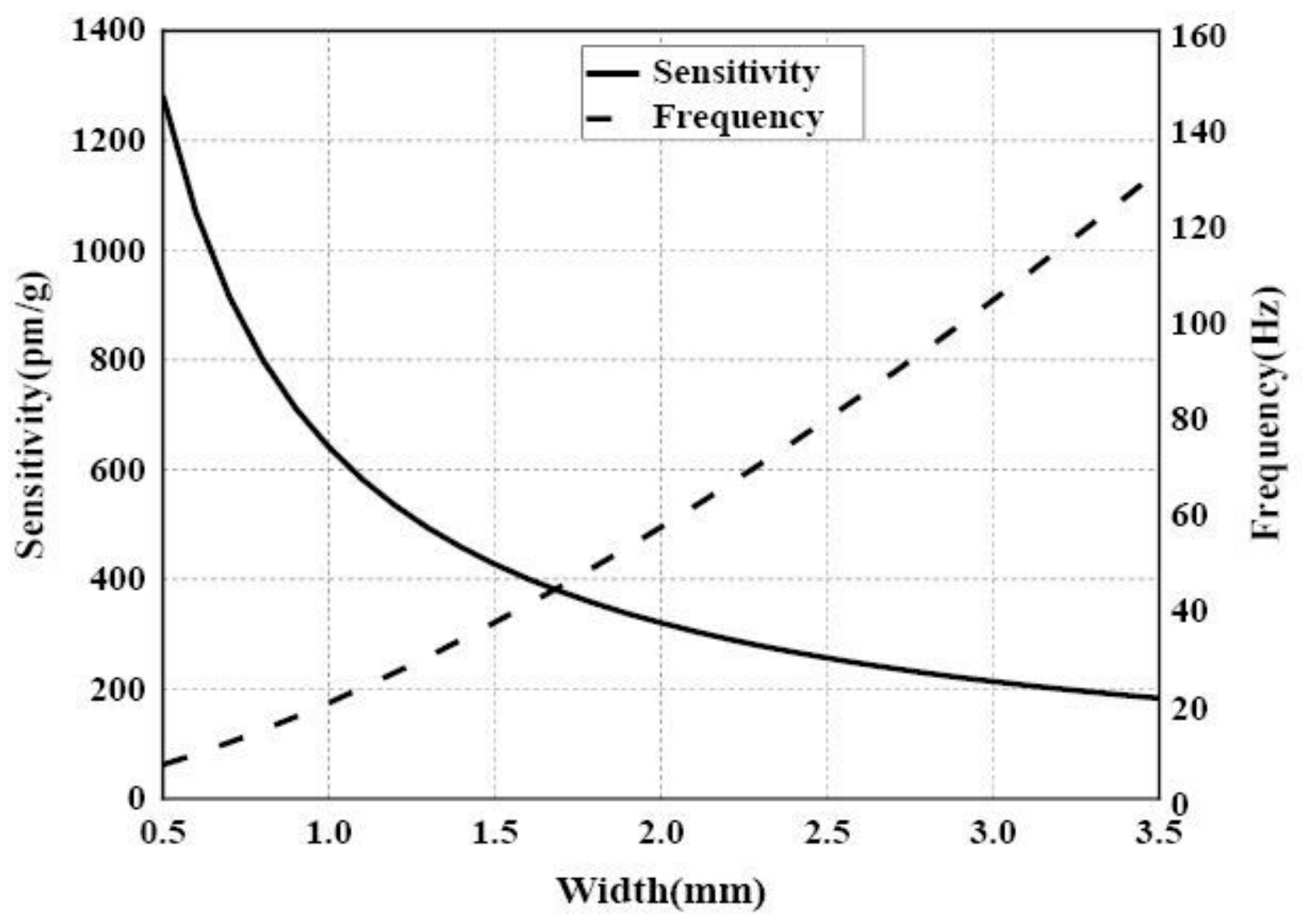

Figure 4

Changes of sensitivity $S$ and resonant frequency $f 0$ with width $b$ 


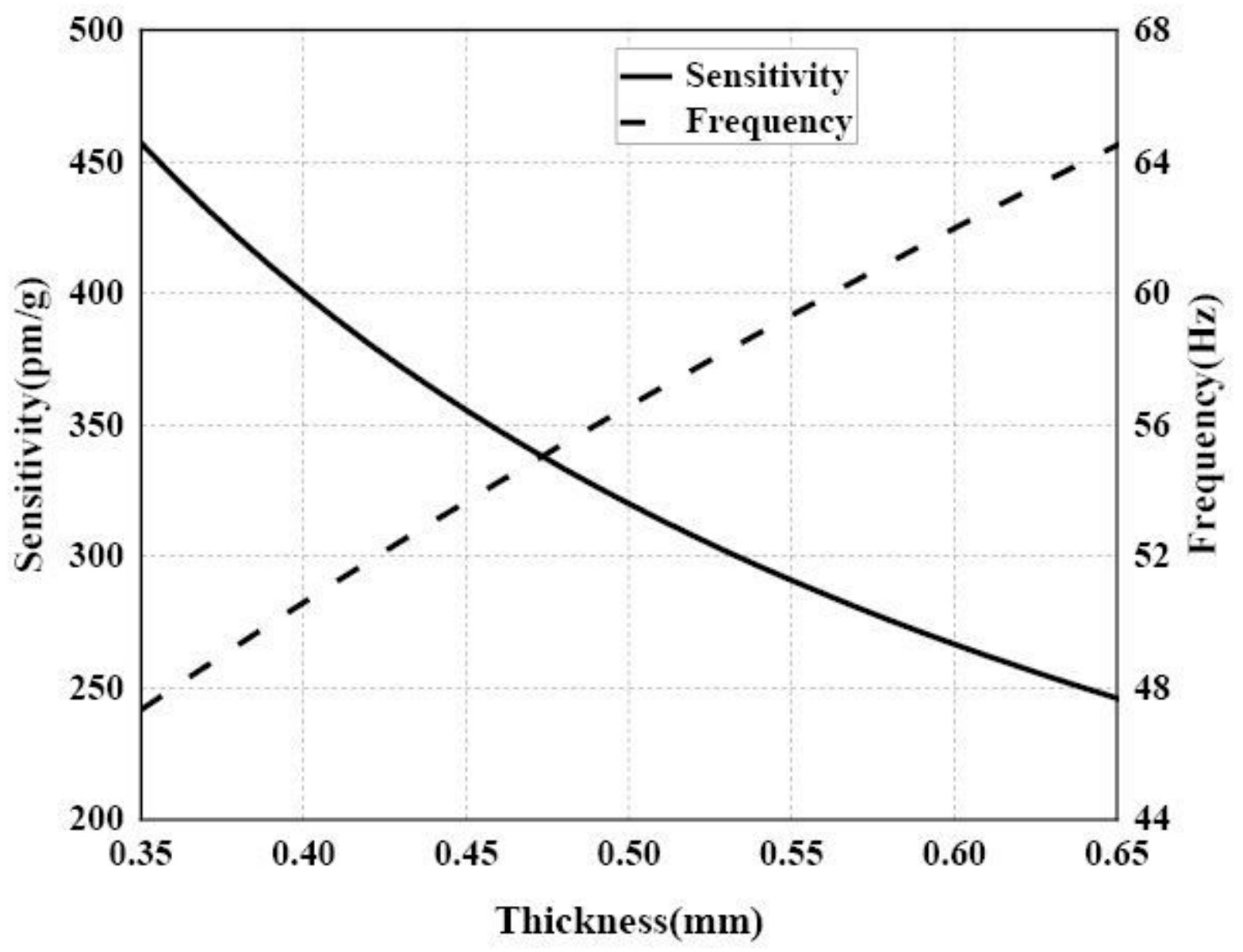

Figure 5

Changes of sensitivity $\mathrm{S}$ and resonant frequency $\mathrm{f} 0$ with thickness $\mathrm{C}$ 


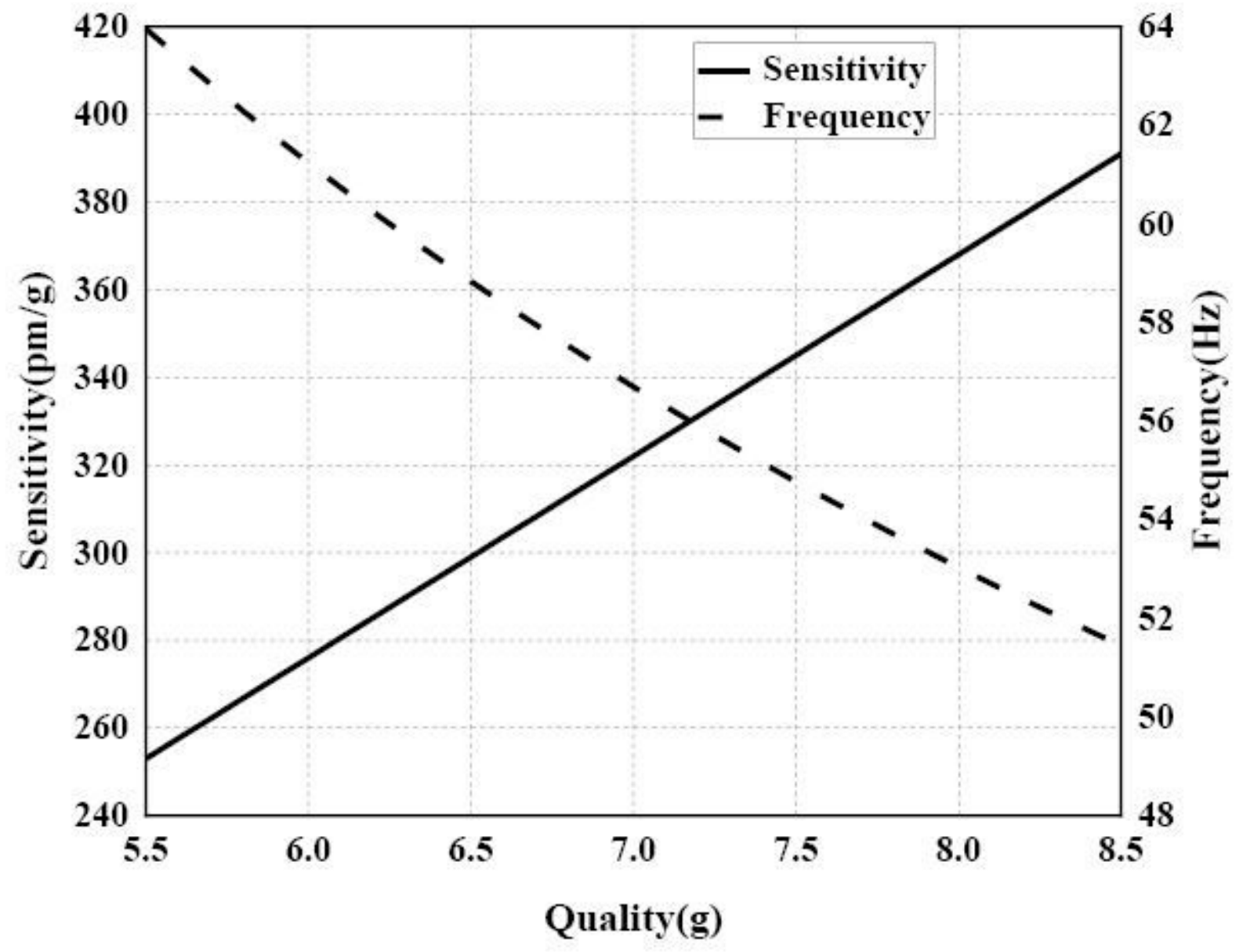

Figure 6

Changes of sensitivity $S$ and resonant frequency $f 0$ with mass $m$
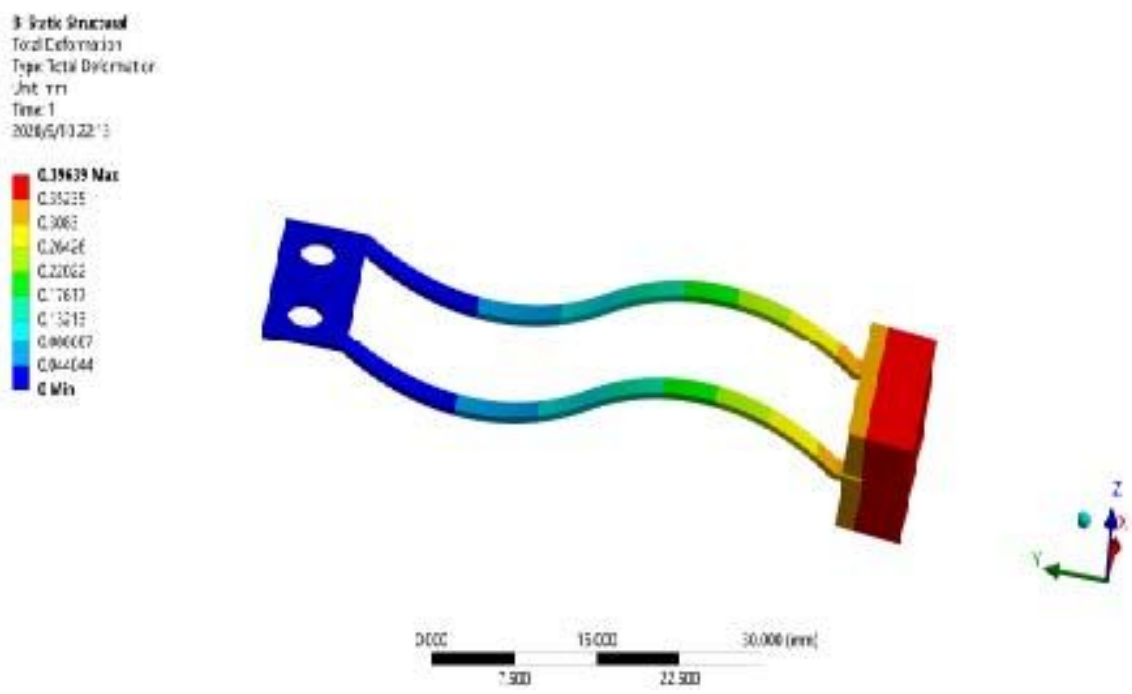

Figure 7 
Static stress simulation analysis

(a) cons

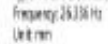

ravanau

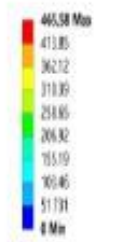

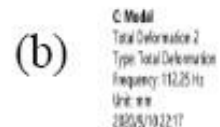

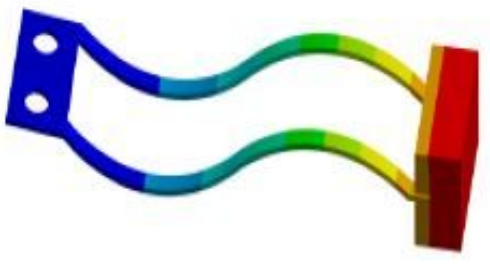

$y^{2}$

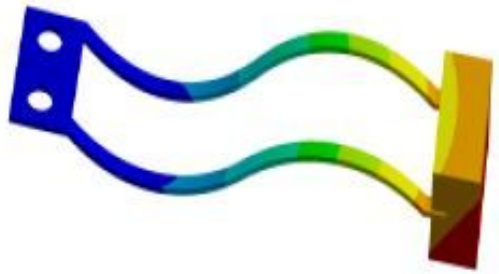

$\int^{2}$

Figure 8

Modal analysis diagrams (a) First-order mode (b) Second-order mode 


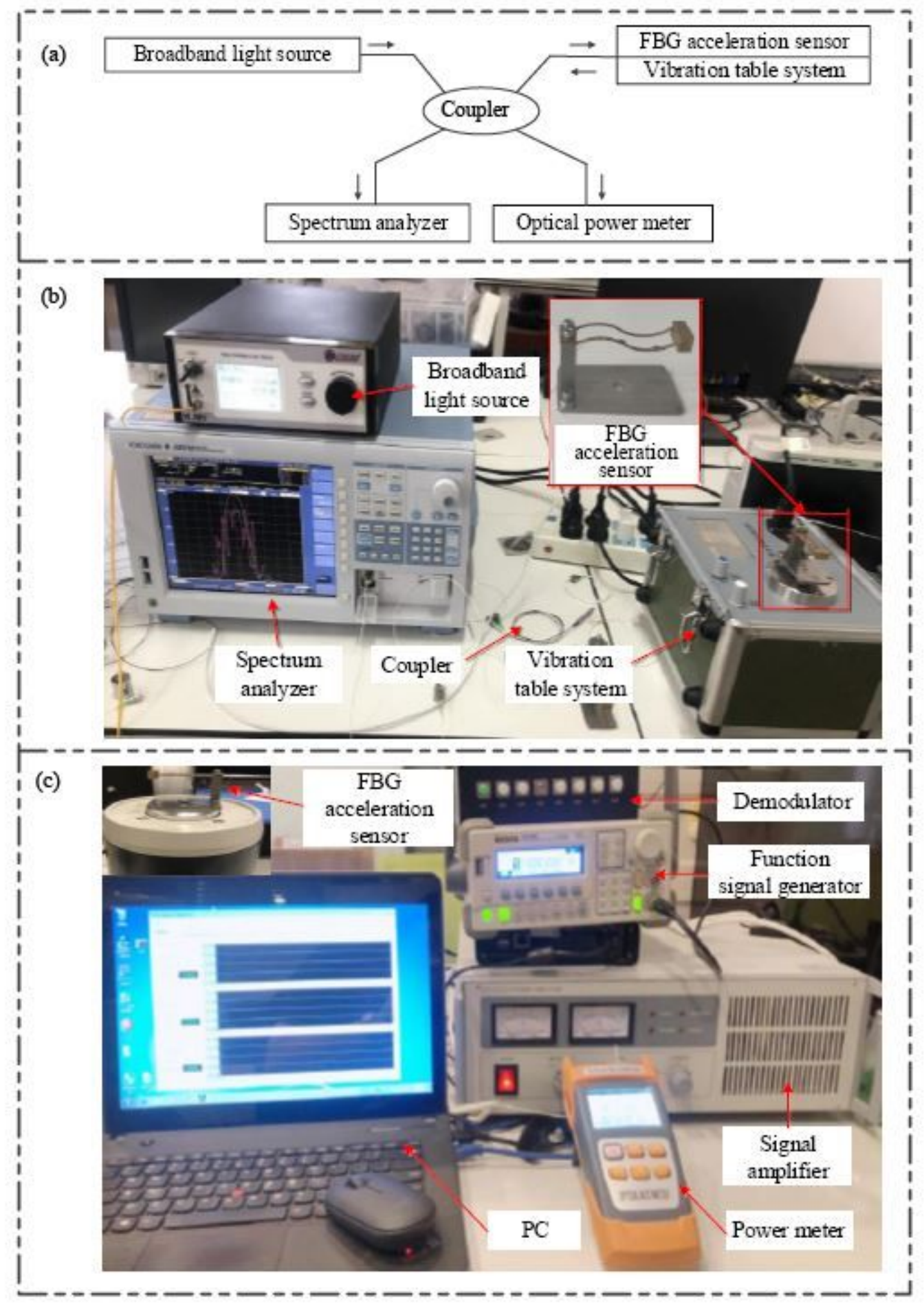

Figure 9

Sensor test system (a) A diagram of the test system (b) Measurement of reflection spectrum bandwidth (c) Measurement of reflected light power 
(a)

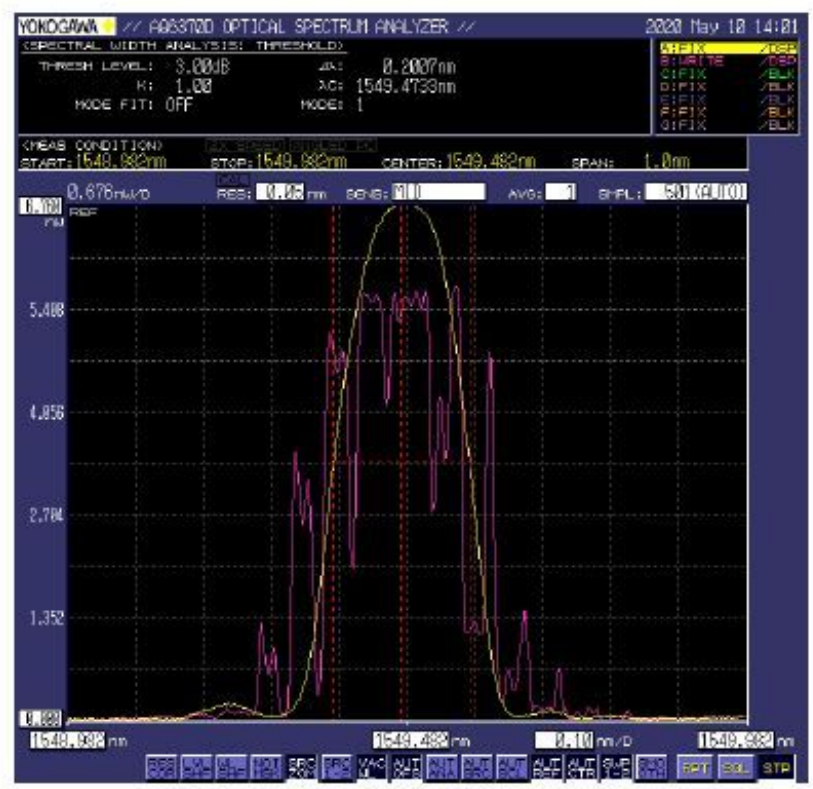

(b)

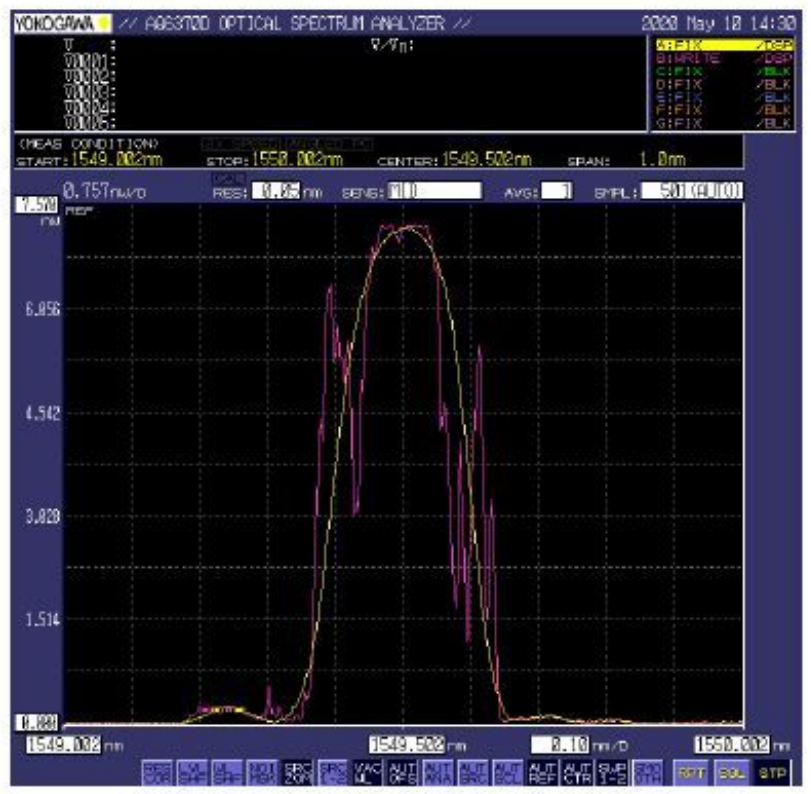

\section{Figure 10}

Reflection spectra under different accelerations: (a) The reflection spectrum bandwidth of the chirp effect produced by the sinusoid-shaped curved beam sensor under the acceleration of about $1 \mathrm{~g}$; (b) The reflection spectrum bandwidth of the chirp effect produced by the rectangular-shaped beam sensor under the acceleration of about $2 \mathrm{~g}$

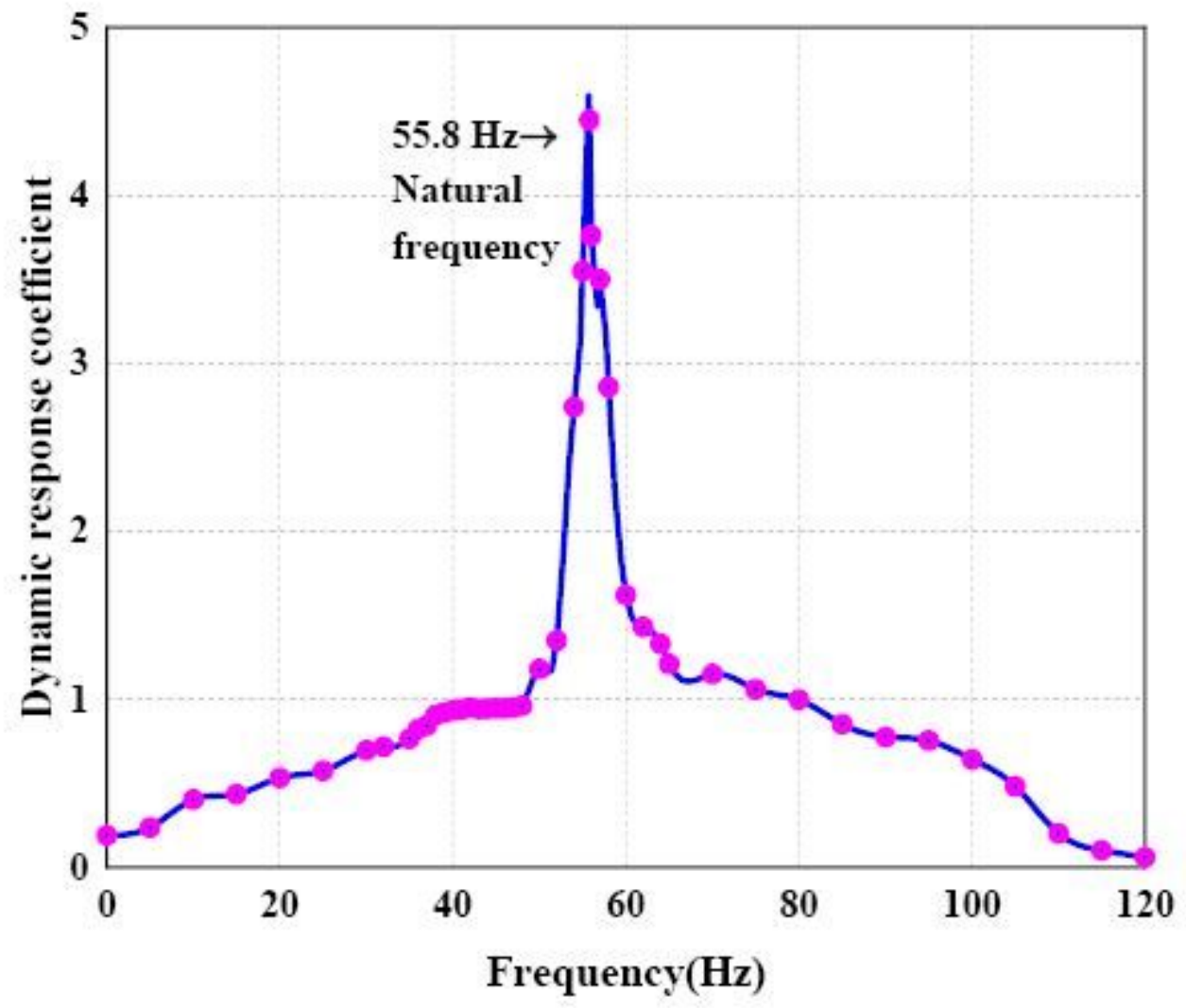




\section{Figure 11}

Acceleration sensitivity amplitude-frequency response curve of the acceleration sensor

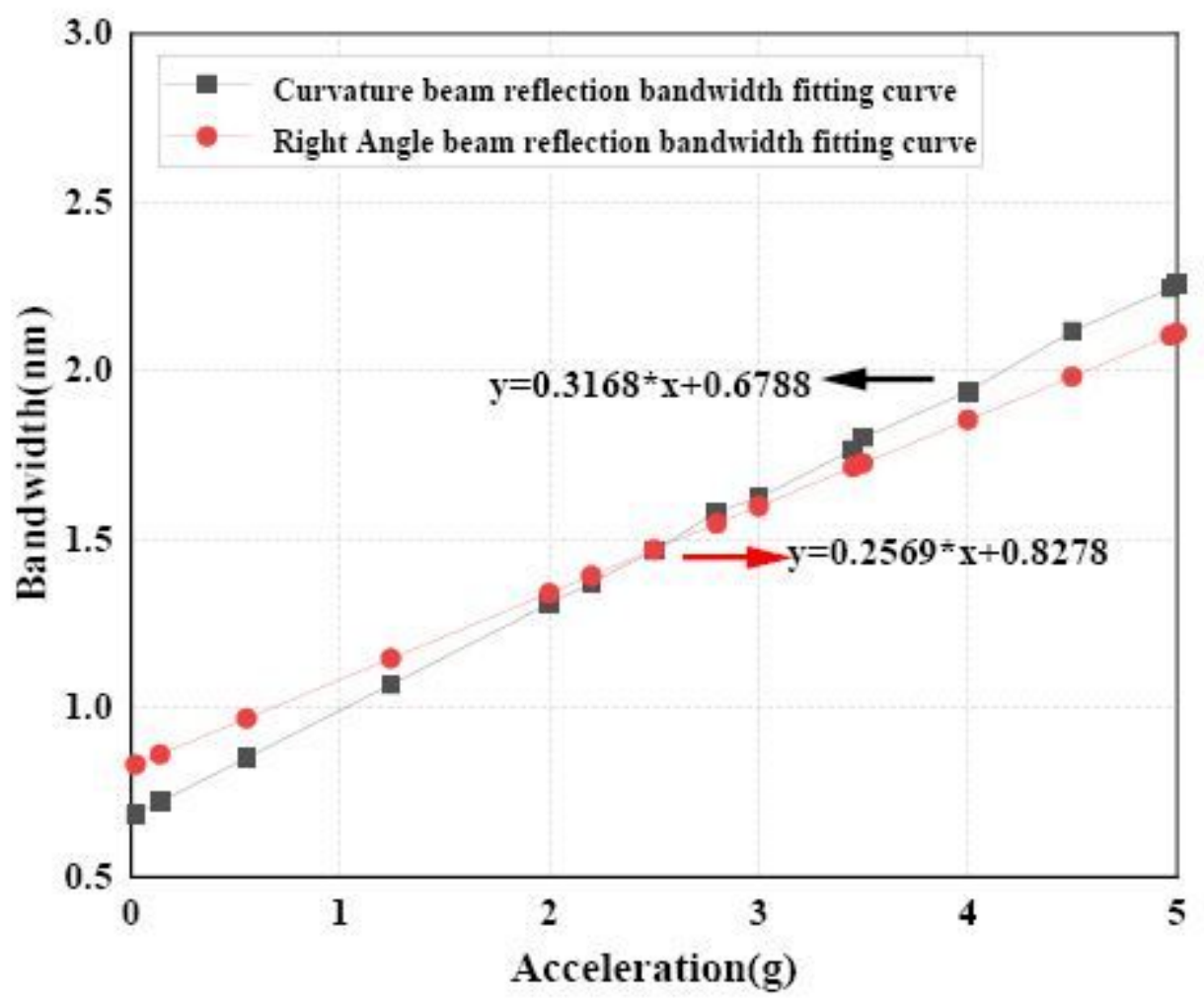

Figure 12

Comparison of reflection spectrum bandwidth fitting curves at two different accelerations 


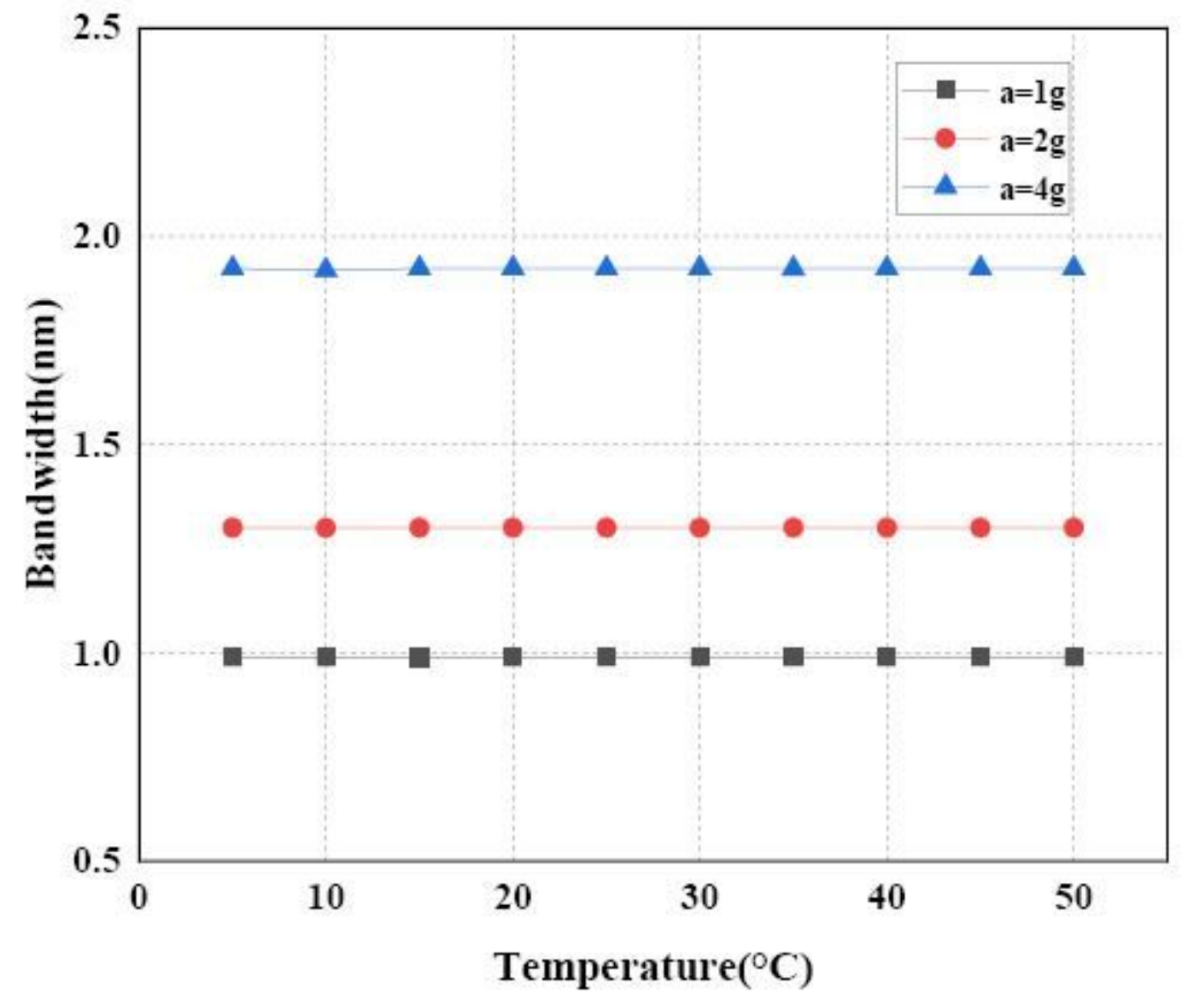

Figure 13

Change of bandwidth with temperature at different accelerations 